\title{
High order sufficient conditions for tracking
}

\author{
M. Barbero-LiñÁn * ${ }^{\dagger}$, M. Sigalotti ${ }^{\ddagger}$ \\ INRIA (Projet CORIDA) and Institut Élie Cartan de Nancy, \\ Université Nancy 1, BP 239, Vandouvre-lès-Nancy, 54506 France.
}

November 30, 2009

\begin{abstract}
In this paper, we study under which conditions the trajectories of a mechanical control system can track any curve on the configuration manifold. We focus on systems that can be represented as forced affine connection control systems and we generalize the sufficient conditions for tracking known in the literature. The sufficient conditions are expressed in terms of convex cones of vector fields defined through particular brackets of the control vector fields of the system. The tracking control laws obtained by our constructions depend on several parameters. By imposing suitable asymptotic conditions on such parameters, we construct algorithmically one-parameter tracking control laws. The theory is supported by examples of control systems associated with elliptic hovercrafts and ellipsoidal submarines.
\end{abstract}

\section{Introduction}

The tracking problem has gained an increasing interest, mainly because of its applications to robot manipulators as for instance to control the position of underwater vehicles [6] and hovercrafts [15]. The tracking problem appears when a particular trajectory has to be followed by a control system, but there is no control law for the control system that makes this trajectory admissible. Then, the best that can be expected is to find a control law, typically oscillatory, that defines a good enough approximation of the target trajectory.

The mathematical background in the tracking problem includes the averaging theory [16] as explained, for instance, in [0]. The averaging techniques transform differential equations difficult to

\footnotetext{
*e-mail: mbarbero@mast.queensu.ca

${ }^{\dagger}$ At present, Department of Mathematics \& Statistics, Queen's University, Kingston (ON), Canada.

${ }^{\ddagger} \mathbf{e}$-mail: mario.sigalotti@inria.fr
}

solve into other differential equations whose solutions approximate fairly well the solutions to the first set of equations. This is useful to approximate solutions to differential equations that depend on time or on parameters.

Differential geometry has provided a suitable framework to study in an intrinsic way typical mechanical control systems in engineering as, for instance, underwater submarines, aircraft models, hovercrafts and so on [5]. Here, we focus on forced affine connection control systems and generalize the sufficient conditions for tracking a trajectory that exist in the literature from a geometric viewpoint [5]. The existent results can be interpreted as first-order sufficient conditions because only the control vector fields and particular brackets, called symmetric products, between them get involved in the statement of the sufficient conditions. However, our conditions need longer symmetric products and so they are said to be of order higher than two.

In [6] it was observed that the tracking is possible for specific underwater vehicles, even though they do not satisfy the geometric sufficient conditions known in the literature. That motivates our research in order to obtain more general geometric sufficient conditions that ensure the tracking property for a wider range of control systems.

The chances to be able to track a target trajectory are related to some controllability requirement and to the avoidance of "bad" directions. In an informal way, these "bad" directions have to be interpreted as directions that will not make possible to have the starting point in the interior of the reachable set. (We refer to [5] for an accurate description of the obstructions to controllability in terms of the symmetric products and of particular vector-valued quadratic forms.) The sufficient conditions for being able to track unfeasible trajectories are also related to how nonholonomicity allows to enlarge the set of admissible velocities for the control system. In this regard, constructions of convex cones [4], the 
above-mentioned vector-valued quadratic forms [5] and some techniques similar to the ones in [12] have been useful for obtaining the constructions considered here.

The paper is organized as follows. Section 2 contains the necessary background in forced affine connection control systems and in chronological calculus [2]. Section 3 defines properly the notion of trackability and reviews the geometric sufficient conditions in the literature 5 . Sections 4 and 5 contain the main contributions of the paper and some examples to justify the utility of these results.

\section{Notation and preliminaries}

Denote by $\mathbb{N}$ the set of positive natural numbers and write $\mathbb{N}_{0}$ for $\mathbb{N} \cup\{0\}$. Fix $n \in \mathbb{N}$. From now on, $Q$ is a $n$-dimensional smooth manifold and $\mathfrak{X}(Q)$ denotes the set of smooth vector fields on $Q$. All the vector fields are considered smooth, unless otherwise stated. Let $\tau_{Q}: T Q \rightarrow Q$ be the canonical tangent projection, a vector field $X$ on $Q$ defined along $\tau_{Q}$ is a mapping $X: T Q \rightarrow T Q$ such that $\tau_{Q} \circ X=\tau_{Q}$.

\subsection{Affine connection control systems}

The trajectories $\gamma: I \rightarrow Q$ of a Lagrangian mechanical systems on a manifold $Q$ are minimizers of the action functional

$$
A_{L}(\gamma)=\int_{I} L(t, \dot{\gamma}(t)) \mathrm{d} t
$$

associated with a Lagrangian function $L: \mathbb{R} \times T Q \rightarrow$ $\mathbb{R}$.

The solutions to this variational problem must satisfy the well-known Euler-Lagrange equations,

$$
\frac{\mathrm{d}}{\mathrm{d} t}\left(\frac{\partial L}{\partial v^{i}}\right)-\frac{\partial L}{\partial q^{i}}=0, \quad i=1, \ldots, n,
$$

where $\left(q^{i}, v^{i}\right)$ are local coordinates for $T Q$. Here we consider controlled Euler-Lagrange equations obtained by modifying the right-hand side on the above equation, as follows:

$$
\frac{\mathrm{d}}{\mathrm{d} t}\left(\frac{\partial L}{\partial v^{i}}\right)-\frac{\partial L}{\partial q^{i}}=\sum_{a=1}^{k} u_{a} Y_{a}^{i}, \quad i=1, \ldots, n,
$$

with $u_{a}: I \rightarrow \mathbb{R}, Y_{a}^{i}: Q \rightarrow \mathbb{R}$.

When the manifold $Q$ is endowed with the Riemannian structure given by a Riemannian metric $g$ and the Lagrangian function $L_{g}\left(v_{q}\right)=\frac{1}{2} g\left(v_{q}, v_{q}\right)$ is considered, the solutions to (11) turn out to be the geodesics of the Levi-Civita affine connection $\nabla^{g}$ associated with the Riemannian metric. (See [5] for more details and for many examples of mechanical control systems that fit in this description.)

When control forces are added to the geodesic equations we obtain an affine connection control system

$$
\nabla_{\dot{\gamma}(t)}^{g} \dot{\gamma}(t)=\sum_{a=1}^{k} u_{a}(t) Y_{a}(\gamma(t)),
$$

with $Y_{a}$ being vector fields on $Q$.

The notion of affine connection control system can be extended without the need of the Levi-Civita connection.

Definition 2.1. An affine connection is a mapping

$$
\begin{aligned}
\nabla: \mathfrak{X}(Q) \times \mathfrak{X}(Q) & \longrightarrow \mathfrak{X}(Q) \\
(X, Y) & \longmapsto \nabla(X, Y)=\nabla_{X} Y,
\end{aligned}
$$

satisfying the following properties:

1. $\nabla$ is $\mathbb{R}$-linear on $X$ and on $Y$;

2. $\nabla_{f X} Y=f \nabla_{X} Y$ for every $f \in \mathcal{C}^{\infty}(Q)$;

3. $\nabla_{X} f Y=f \nabla_{X} Y+(X f) Y$, for every $f \in$ $\mathcal{C}^{\infty}(Q)$. (Here $X f$ denotes the derivative of $f$ in the direction $X$.)

The mapping $\nabla_{X} Y$ is called the covariant derivative of $Y$ with respect to $X$. Given local coordinates $\left(q^{i}\right)$ on $Q$, the Christoffel symbols for the affine connection in these coordinates are given by

$$
\nabla_{\frac{\partial}{\partial q^{j}}} \frac{\partial}{\partial q^{r}}=\sum_{i=1}^{n} \Gamma_{j r}^{i} \frac{\partial}{\partial q^{i}}
$$

From the properties of the affine connection, we have

$$
\nabla_{X} Y=\sum_{i, j, r=1}^{n}\left(X^{j} \frac{\partial Y^{i}}{\partial q^{j}}+\Gamma_{j r}^{i} X^{j} Y^{r}\right) \frac{\partial}{\partial q^{i}},
$$

where $X=\sum_{i=1}^{n} X^{i} \partial / \partial q^{i}$ and $Y=\sum_{i=1}^{n} Y^{i} \partial / \partial q^{i}$.

Definition 2.2. A forced affine connection control system (FACCS) is a control mechanical system given by $\Sigma=(Q, \nabla, Y, \mathscr{Y}, U)$ where

- $Q$ is a smooth $n$-dimensional manifold called the configuration manifold,

- $Y$ is a time-dependent vector field along the projection $\tau_{Q}: T Q \rightarrow Q$, measurable and bounded with respect to the time and affine with respect to the velocities, 
- $\mathscr{Y}$ is a set of $k$ control vector fields on $Q$, and

- $U \subseteq \mathbb{R}^{k}$.

A trajectory $\gamma: I \subset \mathbb{R} \rightarrow Q$ is admissible for $\boldsymbol{\Sigma}$ if $\dot{\gamma}: I \rightarrow T Q$ is absolutely continuous and there exists a measurable and bounded control $u: I \rightarrow U$ such that the dynamical equations of the control system $\Sigma$

$$
\nabla_{\dot{\gamma}(t)} \dot{\gamma}(t)=Y(t, \dot{\gamma}(t))+\sum_{a=1}^{k} u_{a}(t) Y_{a}(\gamma(t))
$$

are fulfilled (almost everywhere).

The vector field $Y$ includes all the noncontrolled external forces; e.g., the potential and the non-potential forces. The assumption that $Y$ is affine with respect to the velocities means that, in every local system of coordinates $\left(q^{i}, v^{i}\right)$ on $T Q$, $Y$ can be written as

$$
Y\left(t, v_{q}\right)=Y_{0}(t, q)+\sum_{i=1}^{n} v^{i} Y^{i}(t, q) .
$$

Equation (2) can be rewritten as a first-order control-affine system on $T Q$,

$\dot{\Upsilon}(t)=Z(\Upsilon(t))+Y^{V}(t, \Upsilon(t))+\sum_{a=1}^{k} u_{a}(t) Y_{a}^{V}(\Upsilon(t))$

where $\Upsilon: I \rightarrow T Q$ is such that $\tau_{Q} \circ \Upsilon=\gamma, Z$ is the geodesic spray associated to the affine connection on $Q$ and $Y_{a}^{V}$ denotes the vertical lift of the vector field $Y_{a}$ (see [1] for more details).

Apart from the usual Lie bracket that provides $\mathfrak{X}(Q)$ with a Lie algebra structure, the following product of elements in $\mathfrak{X}(Q)$ associated to $\nabla$ can be introduced.

Definition 2.3. The symmetric product is the map

$$
\begin{aligned}
\langle\cdot: \cdot\rangle: \mathfrak{X}(Q) \times \mathfrak{X}(Q) & \longrightarrow \mathfrak{X}(Q) \\
(X, Y) & \longmapsto \nabla_{X} Y+\nabla_{Y} X .
\end{aligned}
$$

It can be proved that

$$
\left[Y_{a}^{V},\left[Z, Y_{b}^{V}\right]\right]=\left\langle Y_{a}: Y_{b}\right\rangle^{V}
$$

(see [司]).

\subsection{Chronological calculus}

We recall in this section some notion of chronological calculus, which is used later as a tool in the study of the asymptotic behavior of endpoint mappings depending on parameters. For a comprehensive discussion and for the proofs of all results stated in this section see [2]. In the sequel all vector fields, autonomous and non-autonomous, are assumed to be complete. The behavior of non-complete vector fields on compact sets can be studied by considering suitable cut-off procedures.

Given a non-autonomous vector field $X_{\tau}$ on some manifold $M$, where $\tau$ denotes the time variable and the map $(\tau, q) \mapsto X_{\tau}(q)$ is assumed to be smooth with respect to $q$ and measurable bounded with respect to $\tau$, we denote by $\overrightarrow{\exp } \int_{0}^{t} X_{\tau} \mathrm{d} \tau$ the diffeomorphism of $M$ onto itself corresponding to the flow from time 0 to time $t$ of $X_{\tau}$. Hence, $\overrightarrow{\exp } \int_{0}^{t} X_{\tau} \mathrm{d} \tau(\bar{q})$ is the evaluation at time $t$ of the solution to the non-autonomous Cauchy problem

$$
\dot{q}(\tau)=X_{\tau}(q(\tau)), \quad q(0)=\bar{q} .
$$

Any diffeomorphism $P: M \rightarrow M$ defines an isomorphism $\operatorname{Ad} P$ of $\mathfrak{X}(M)$ through the rule

$$
\operatorname{Ad} P(Y)(q)=P_{*}\left(Y\left(P^{-1}(q)\right)\right),
$$

where $P_{*}$ denotes the pushforward by $P$.

If $P=\overrightarrow{\exp } \int_{0}^{t} X_{\tau} \mathrm{d} \tau$, then we write

$$
\operatorname{Ad} P=\overrightarrow{\exp } \int_{0}^{t} \operatorname{ad} X_{\tau} \mathrm{d} \tau
$$

If $\operatorname{ad}^{(m)} X_{\tau}(Y)=0$ for some $m \in \mathbb{N}$ and every $\tau$, then

$$
\begin{aligned}
& \overrightarrow{\exp } \int_{0}^{t} \operatorname{ad} X_{\tau} \mathrm{d} \tau(Y)=Y+\int_{0}^{t}\left[X_{\tau}, Y\right] \mathrm{d} \tau \\
& \quad+\int_{0}^{t} \int_{0}^{\tau_{1}}\left[X_{\tau_{2}},\left[X_{\tau_{1}}, Y\right] \mathrm{d} \tau_{2} \mathrm{~d} \tau_{1}+\cdots+\right. \\
& \int_{0}^{t} \int_{0}^{\tau_{1}} \cdots \int_{0}^{\tau_{\tau_{m-2}}} \operatorname{ad} X_{\tau_{m-1}} \circ \cdots \circ \operatorname{ad} X_{\tau_{1}} Y \mathrm{~d} \tau_{m-1} \cdots \mathrm{d} \tau_{1} .
\end{aligned}
$$

In the framework of chronological calculus the flow of the sum of two non-autonomous vector fields can be conveniently represented by the following variation formula

$$
\begin{aligned}
& \overrightarrow{\exp } \int_{0}^{t}\left(X_{\tau}+Y_{\tau}\right) \mathrm{d} \tau= \\
& \overrightarrow{\exp } \int_{0}^{t} X_{\tau} \mathrm{d} \tau \circ \overrightarrow{\exp } \int_{0}^{t}\left(\overrightarrow{\exp } \int_{0}^{\tau} \operatorname{ad} X_{s} \mathrm{~d} s\right) Y_{\tau} \mathrm{d} \tau \text {. }
\end{aligned}
$$

Let us recall a useful result for the convergence of flows of non-autonomous vector fields. It states, roughly speaking, that the flows converge if the vector fields converge in integral sense. For further results and a discussion on this kind of convergence from the point of view of ordinary differential equations and control theory, see [3, 11]. 
Lemma 2.4. 粵, Lemma 8.10] Let $Z_{\tau}^{j}, j \in \mathbb{N}$, and $Z_{\tau}, \tau \in\left[0, t_{1}\right]$, be non-autonomous vector fields on $M$, bounded with respect to $\tau$, and let these vector fields have a compact support. If

$$
\int_{0}^{t} Z_{\tau}^{j} \mathrm{~d} \tau \rightarrow \int_{0}^{t} Z_{\tau} \mathrm{d} \tau, \quad j \rightarrow \infty
$$

then

$$
\overrightarrow{\exp } \int_{0}^{t} Z_{\tau}^{j} \mathrm{~d} \tau \rightarrow \overrightarrow{\exp } \int_{0}^{t} Z_{\tau} \mathrm{d} \tau, \quad j \rightarrow \infty
$$

both convergences being uniform with respect to $(t, q) \in\left[0, t_{1}\right] \times M$ and uniform with all derivatives with respect to $q \in M$.

Another, even more standard, result on the convergence of flows is the following, that we find useful to state as a separate lemma. Its difference from the previous one can be described as follows: if the dependence on time of the $Z_{\tau}^{j}$ is prescribed independently on $j$ (it would be enough that they converge uniformly), then the uniform convergence of flows is guaranteed by the uniform convergence of the vector fields, even without any knowledge about the convergence of their derivatives with respect to the state variables. For completeness, we provide a brief proof for it.

Lemma 2.5. Let $\lambda_{1}, \ldots, \lambda_{m} \in L^{\infty}\left(\left[0, t_{1}\right], \mathbb{R}\right)$. Let $Z_{\tau}^{j}, n \in \mathbb{N}$, and $Z_{\tau}, \tau \in\left[0, t_{1}\right]$, be non-autonomous vector fields on $M$ of the form $Z_{\tau}^{j}=\lambda_{1}(\tau) Z_{1}^{j}+\cdots+$ $\lambda_{m}(\tau) Z_{m}^{j}$ and $Z_{\tau}=\lambda_{1}(\tau) Z_{1}+\cdots+\lambda_{m}(\tau) Z_{m}$, with $Z_{i}^{j}, Z_{i} \in \mathfrak{X}(M)$ with compact support. If $Z_{i}^{j} \rightarrow Z_{i}$, $j \rightarrow \infty$, then

$$
\overrightarrow{\exp } \int_{0}^{t} Z_{\tau}^{j} \mathrm{~d} \tau \rightarrow \overrightarrow{\exp } \int_{0}^{t} Z_{\tau} \mathrm{d} \tau, \quad j \rightarrow \infty
$$

both convergences being uniform (the first with respect to the state $q$ and the second with respect to $(t, q))$.

Proof. Using the variation formula (6) with $X_{\tau}=$ $Z_{\tau}$ and $Y_{\tau}=Z_{\tau}^{j}-Z_{\tau}$, we get

$$
\begin{aligned}
& \overrightarrow{\exp } \int_{0}^{t} Z_{\tau}^{j} \mathrm{~d} \tau=\overrightarrow{\exp } \int_{0}^{t} Z_{\tau} \mathrm{d} \tau \circ \\
& \overrightarrow{\exp } \int_{0}^{t}\left(\overrightarrow{\exp } \int_{0}^{\tau} \operatorname{ad} Z_{s} \mathrm{~d} s\right)\left(Z_{\tau}^{j}-Z_{\tau}\right) \mathrm{d} \tau .
\end{aligned}
$$

Since $\left(\overrightarrow{\exp } \int_{0}^{\tau} Z_{s} \mathrm{~d} s\right)_{*}, \tau \in\left[0, t_{1}\right]$, is a compact family of operators, then the last diffeomorphism in (7) converges uniformly to the identity for $t \in\left[0, t_{1}\right]$ as $j$ goes to infinity.

\section{Tracking problem}

The need of trackability appears when one tries to follow a particular trajectory on the configuration manifold, called reference or target trajectory, which is not a solution of the FACCS considered. A trajectory is successfully tracked if there exist solutions to the FACCS that approximate it arbitrarily well.

Consider any distance d: $Q \times Q \rightarrow \mathbb{R}$ on $Q$ whose corresponding metric topology coincides with the topology on $Q$. From now on $I$ will denote the interval $\left[0, t_{1}\right]$, with $t_{1}>0$.

Definition 3.1. A control system $\Sigma$ is trackable if for every continuous curve $\gamma: I \rightarrow Q$, for every $v \in T_{\gamma(0)} Q$ and for every strictly positive tolerance $\epsilon$, there exist a control $u^{\epsilon} \in L^{\infty}(I, U)$ such that the solution $\xi^{\epsilon}: I \rightarrow Q$ to $\Sigma$ corresponding to $u^{\epsilon}$ and with initial condition $\dot{\xi}^{\epsilon}(0)=v$ verifies

$$
\mathrm{d}\left(\gamma(t), \xi^{\epsilon}(t)\right)<\epsilon
$$

for every time $t \in I$.

Remark 3.2. Since any continuous curve can be uniformly approximated, with arbitrary precision, by a smooth curve having a prescribed tangent vector at its initial point, then $\Sigma$ is trackable if and only if every curve $\gamma: I \rightarrow Q$ on $Q$ of class $\mathcal{C}^{\infty}$ is trackable for $\Sigma$, i.e., for every $\epsilon>0$, there exist $u^{\epsilon} \in L^{\infty}(I, U)$ such that the solution $\xi^{\epsilon}: I \rightarrow Q$ to $\Sigma$ corresponding to $u^{\epsilon}$ and with initial condition $\dot{\xi}^{\epsilon}(0)=\dot{\gamma}(0)$ verifies $\mathrm{d}\left(\gamma(t), \xi^{\epsilon}(t)\right)<\epsilon$ for every $t \in I$.

In order to give some insights into particular sufficient conditions for tracking, we are going to review a result in the literature.

Theorem 3.3. [5, Theorem 12.26] Let $\Sigma=$ $(Q, \nabla, Y, \mathscr{Y}, U)$ be a FACCS such that

- the distribution generated by $\mathscr{Y}=\left\{Y_{1}, \ldots, Y_{k}\right\}$ is regular, that is, it is a subbundle of $T Q$;

- $\left\langle Y_{a}: Y_{a}\right\rangle \in \operatorname{span}_{\mathcal{C}^{\infty}(Q)} \mathscr{Y}$ for every a $\in$ $\{1, \ldots, k\}$, i.e., $\left\langle Y_{a}: Y_{a}\right\rangle=\sum_{b=1}^{k} \sigma_{a b} Y_{b}$, where $\sigma_{a b} \in \mathcal{C}^{\infty}(Q)$;

- the distribution $\operatorname{Sym}^{(1)}(\mathscr{Y}) \quad d e$ fined by $\operatorname{Sym}^{(1)}(\mathscr{Y})_{q}=\operatorname{span}_{\mathbb{R}} \mathscr{Y}_{q}+$ $\operatorname{span}_{\mathbb{R}}\{\langle W: Z\rangle(q) \mid W, Z \in \mathscr{Y}\}$ is the entire tangent bundle $T Q$.

Let $\gamma_{\mathrm{ref}}: I \rightarrow Q$ be a reference trajectory of class $\mathcal{C}^{3}$. Define the functions $u_{\mathrm{ref}, a}, u_{\mathrm{ref}, b c}: I \rightarrow \mathbb{R}$, 
$a, b, c \in\{1, \ldots, k\}, b<c$, as solutions of class $\mathcal{C}^{1}$ to

$$
\begin{aligned}
& \nabla_{\dot{\gamma}_{\mathrm{ref}}(t)} \dot{\gamma}_{\mathrm{ref}}(t)-Y\left(t, \dot{\gamma}_{\mathrm{ref}}(t)\right) \\
& =\sum_{a=1}^{k} u_{\mathrm{ref}, a}(t) Y_{a}\left(\gamma_{\mathrm{ref}}(t)\right) \\
& +\sum_{b, c=1, b<c}^{k} u_{\mathrm{ref}, b c}(t)\left\langle Y_{b}: Y_{c}\right\rangle\left(\gamma_{\mathrm{ref}}(t)\right) .
\end{aligned}
$$
$\mathbb{R}^{k}$ by

Define $u_{\text {slow }}: I \times T Q \rightarrow \mathbb{R}^{k}, u_{\mathrm{osc}}: \mathbb{R} \times I \times T Q \rightarrow$

$$
\begin{aligned}
& u_{\text {slow }, a}\left(t, v_{q}\right)=u_{\mathrm{ref}, a}(t)+ \\
& \sum_{b=1}^{k}\left(b-1+\sum_{c=b+1}^{k} \frac{\left(u_{\mathrm{ref}, b c}(t)\right)^{2}}{4}\right) \sigma_{a b}(q), \\
& u_{\mathrm{osc}, a}\left(\tau, t, v_{q}\right)=\sum_{c=1}^{a-1} \varphi_{\mathrm{lo}(c, a)}(\tau) \\
& -\frac{1}{2} \sum_{c=a+1}^{k} u_{\mathrm{ref}, a c}(t) \varphi_{\mathrm{lo}(a, c)}(\tau),
\end{aligned}
$$

where $\operatorname{lo}(a, b)=\sum_{j=1}^{a-1}(k-j)+(b-a)$ for $(a, b) \in$ $\{1, \ldots, k\}^{2}, a<b$, and for $i \in \mathbb{N}$

$$
\varphi_{i}: \mathbb{R} \rightarrow \mathbb{R}, \quad t \mapsto \frac{4 \pi i}{T} \cos \left(\frac{2 \pi i}{T} t\right) .
$$

Then $\gamma_{\mathrm{ref}}$ is trackable for $\Sigma$ and moreover the solutions $\xi^{\epsilon}$ of $\Sigma, \epsilon>0$, corresponding to the controls

$$
\begin{aligned}
u^{\epsilon}: \mathbb{R} \times T Q & \longrightarrow U \subset \mathbb{R}^{k} \\
\left(t, v_{q}\right) & \longmapsto u_{\text {slow }}\left(t, v_{q}\right)+\frac{1}{\epsilon} u_{\text {osc }}\left(\frac{t}{\epsilon}, t, v_{q}\right)
\end{aligned}
$$

with initial condition $\dot{\xi}^{\epsilon}(0)=\dot{\gamma}_{\text {ref }}(0)$ are such that $\mathrm{d}\left(\gamma_{\mathrm{ref}}(t), \xi^{\epsilon}(t)\right)$ tends to zero as $\epsilon$ goes to zero uniformly with respect to $t \in I$.

Remark 3.4. Observe that under the hypotheses of Theorem 3.3 not only the tracking is guaranteed, but also the tracking control law is given explicitly.

\section{A more general tracking result}

The idea of the following construction is to identify, given the set $\mathscr{Y}$, a larger set of control vector fields $\mathscr{K}_{1}$ such that every trajectory solution of the FACCS obtained by replacing $\operatorname{span}_{\mathcal{C}^{\infty}(Q)} \mathscr{Y}$ by $\mathscr{K}_{1}$ can be tracked by solutions of the original FACCS $\Sigma$. Repeating the construction on $\mathscr{K}_{1}$ we obtain an even larger family $\mathscr{K}_{2}$ and so on. If eventually $\mathscr{K}_{l}(q)=T_{q} Q$ for every $q \in Q$ for some $l \in \mathbb{N}$, then we can show that the system is trackable.
In order to generalize the sufficient conditions for tracking given in Theorem 3.3, we construct the following set of vector fields on $Q$ :

$$
\begin{aligned}
\mathscr{K}_{0} & =\overline{\operatorname{span}_{\mathcal{C}^{\infty}(Q)} \mathscr{Y}}, \\
\mathscr{K}_{l} & =\overline{\mathscr{K}_{l-1}-\operatorname{co}\left\{\langle Z: Z\rangle \mid Z \in \mathrm{L}\left(\mathscr{K}_{l-1}\right)\right\}},
\end{aligned}
$$

for $l \in \mathbb{N}$, where, for $A \subset \mathfrak{X}(Q), \mathrm{L}(A)=A \cap(-A)$, $\operatorname{co}(A)$ denotes the convex hull of $A$, and $\bar{A}$ is the closure of $A$ in $\mathfrak{X}(Q)$ with respect to the topology of the uniform convergence on compact sets. For $A \subset \mathfrak{X}(Q)$ we also write

$$
A(q)=\{Y(q) \mid Y \in A\} \subseteq T_{q} Q .
$$

Proposition 4.1. For every $l \in \mathbb{N}_{0}$, $\mathscr{K}_{l}$ is a convex cone of $\mathscr{X}(Q)$. In particular, for every $q \in Q, \mathscr{K}_{l}(q)$ is a convex cone with vertex at $0 \in T_{q} Q$.

Proof. The proposition is proved by induction having in mind that the set $\mathscr{K}_{l}$ is a convex cone if it contains all conic combinations of elements of $\mathscr{K}_{l}$. Remember that a conic combination of elements of $\mathscr{K}_{l}$ is of the form $\lambda_{1} W_{1}+\cdots+\lambda_{r} W_{r}$ with $\lambda_{i} \in \mathbb{R}_{\geq 0}$, $W_{i} \in \mathscr{K}_{l}$ for every $i \in\{1, \ldots, r\}$.

First, $\mathscr{K}_{0}$ is subspace of $\mathscr{X}(Q)$ and thus it is a convex cone.

The induction step consists of proving that if $\mathscr{K}_{i}$ is a convex cone, so is $\mathscr{K}_{i+1}$. First notice that, if $W \in L\left(\mathscr{K}_{i}\right)$ and $\lambda \in \mathbb{R}$, then, by the induction hypothesis, $\lambda W \in L\left(\mathscr{K}_{i}\right)$ and thus $\lambda^{2}\langle W: W\rangle$ belongs to $\left\{\langle Z: Z\rangle \mid Z \in L\left(\mathscr{K}_{i}\right)\right\}$. Hence, $\mathscr{K}_{i+1}$ is the closure of the sum of two convex sets invariant by multiplication by any non-negative scalar. Therefore, $\mathscr{K}_{i+1}$ is itself a convex cone.

Given two functions $\alpha, \beta:[0, T] \rightarrow \mathbb{R}$, let

$$
\Lambda_{T}(\alpha, \beta)=\frac{1}{2 T} \int_{0}^{T}\left(\int_{0}^{\tau} \alpha(s) \mathrm{d} s\right)\left(\int_{0}^{\tau} \beta(s) \mathrm{d} s\right) \mathrm{d} \tau .
$$

We say that a sequence of smooth $T$-periodic functions $\psi_{j}: \mathbb{R} \rightarrow \mathbb{R}, j \in \mathbb{N}$, is $\Lambda_{T}$-orthonormal and zero-mean if $\int_{0}^{T} \psi_{j}(\tau)=0$ for every $j \in \mathbb{N}$ and $\Lambda_{T}\left(\psi_{j}, \psi_{m}\right)=\delta_{j m}$ for $j, m \in \mathbb{N}$, where $\delta_{j m}$ denotes the Kronecker delta.

For instance, the sequence defined in (8), is $\Lambda_{T^{-}}$ orthonormal and zero-mean.

Proposition 4.2. Let $\Sigma=\left(Q, \nabla, Y, \mathscr{Y}, \mathbb{R}^{k}\right)$ be a FACCS and fix $v_{q} \in T Q, T>0, n_{1}, \ldots, n_{k} \in \mathbb{N}$, and $w=\left(w_{1}, \ldots, w_{k}\right) \in L^{\infty}\left(I, \mathbb{R}^{k}\right)$. For every $\epsilon>0$ denote by $\xi^{\epsilon}: I \rightarrow Q$ the solution of

$$
\begin{aligned}
\nabla_{\dot{\xi}^{\epsilon}(t)} \dot{\xi}^{\epsilon}(t) & =Y\left(t, \dot{\xi}^{\epsilon}(t)\right) \\
& +\sum_{a=1}^{k} \frac{1}{\epsilon} \psi_{n_{a}}\left(\frac{t}{\epsilon}\right) w_{a}(t) Y_{a}\left(\xi^{\epsilon}(t)\right),
\end{aligned}
$$


with initial condition $\dot{\xi}^{\epsilon}(0)=v_{q}$, where $\left(\psi_{j}\right)_{j \in \mathbb{N}}$ is a $\Lambda_{T}$-orthonormal and zero-mean sequence. Let also $\gamma: I \rightarrow Q$ be the solution of

$$
\begin{aligned}
& \nabla_{\dot{\gamma}(t)} \dot{\gamma}(t)=Y(t, \dot{\gamma}(t)) \\
& -\sum_{a, b=1}^{k} \delta_{n_{a} n_{b}} w_{a}(t) w_{b}(t)\left\langle Y_{a}: Y_{b}\right\rangle(\gamma(t)),
\end{aligned}
$$

with initial condition $\dot{\gamma}(0)=v_{q}$. Then there exist $C, \epsilon_{0}>0$ such that $\mathrm{d}\left(\gamma(t), \xi^{\epsilon}(t)\right)<C \epsilon$ for every $t \in I$ and every $\epsilon \in\left(0, \epsilon_{0}\right)$.

Proof. The proof of this proposition follows from Theorem 9.32 in [5] by considering the function $u^{a}(t / \epsilon, t)$ that appears there as $\psi_{n_{a}}(t / \epsilon) w_{a}(t)$.

Remark 4.3. Theorem 3.3 follows directly from Proposition 4.2 (and is obtained as such in [5]). Therefore, some of the hypotheses of Theorem 3.3 can easily be relaxed: first of all the distribution generated by $\mathscr{Y}$ need not be a subbundle of $T Q$. Moreover, the hypothesis that $\operatorname{Sym}^{(1)}(\mathscr{Y})=T Q$ can be replaced by the requirement that

$$
\nabla_{\dot{\gamma}_{\text {ref }}(t)} \dot{\gamma}_{\text {ref }}(t)-Y\left(t, \dot{\gamma}_{\text {ref }}(t)\right) \in \operatorname{Sym}^{(1)}(\mathscr{Y})_{\gamma_{\text {ref }}(t)}
$$

for every $t \in I$.

Theorem 4.4. Let $\Sigma=\left(Q, \nabla, Y, \mathscr{Y}, \mathbb{R}^{k}\right)$ be a FACCS. Fix a reference trajectory $\gamma_{\text {ref }}: I \rightarrow Q$ of class $\mathcal{C}^{\infty}$. If for every $t \in I$ there exists $l \in \mathbb{N}$ such that $\mathscr{K}_{l}\left(\gamma_{\text {ref }}(t)\right)=T_{\gamma_{\text {ref }}(t)} Q$, then $\gamma_{\text {ref }}$ is trackable. Therefore, if for every $q \in Q$ there exists $l \in \mathbb{N}$ such that $\mathscr{K}_{l}(q)=T_{q} Q$, then the control system $\Sigma$ is trackable.

Proof. First of all, notice that the last part of the statement (the trackability of $\Sigma$ ) directly follows from the first one (the trackability of $\gamma_{\text {ref }}$ ), which is proved below.

As the reference trajectory $\gamma_{\text {ref }}$ is defined on a compact set and $\gamma_{\text {ref }}$ is continuous, then $\operatorname{Im} \gamma_{\text {ref }}$ is a closed compact set. By hypothesis, for every $t \in I$ there exists $l \in \mathbb{N}$ such that $\mathscr{K}_{l}\left(\gamma_{\text {ref }}(t)\right)=T_{\gamma_{\text {ref }}(t)} Q$. So there exist $n+1$ vector fields in $\mathscr{K}_{l}$ whose conic combinations at $\gamma_{\mathrm{ref}}(t)$ give the whole tangent space $T_{\gamma_{\mathrm{ref}}(t)} Q$. The smoothness of these vector fields guarantees that this is still true in an open neighborhood $U_{t}$ of $\gamma_{\text {ref }}(t)$. In this way we construct an open cover of $\operatorname{Im} \gamma_{\text {ref }}$. As $\operatorname{Im} \gamma_{\text {ref }}$ is compact, there exists a finite open subcover of $\left\{U_{t}\right\}_{t \in I}$ given by $U_{t_{1}}, \ldots, U_{t_{r}}$. For each $t_{i}$ there exists a different $l_{i} \in \mathbb{N}$ such that $\mathscr{K}_{l_{i}}\left(\gamma_{\mathrm{ref}}(t)\right)=T_{\gamma_{\mathrm{ref}}(t)} Q$ for every $t \in \gamma_{\text {ref }}^{-1}\left(U_{t_{i}}\right)$. Then $l=\max \left\{l_{1}, \ldots, l_{r}\right\}$ satisfies $\mathscr{K}_{l}\left(\gamma_{\text {ref }}(t)\right)=T_{\gamma_{\text {ref }}(t)} Q$ for every $t \in I$.
Moreover, there exists a partition of unity subordinated to the finite open subcover $\left\{U_{t_{i}}\right\}_{i=1, \ldots, r}$ (see [10]) that allows us to define a finite set of smooth global vector fields $Z_{a}$ in $\mathscr{K}_{l}$ such that

$$
\nabla_{\dot{\gamma}_{\text {ref }}(t)} \dot{\gamma}_{\text {ref }}(t)-Y\left(t, \dot{\gamma}_{\text {ref }}(t)\right)=\sum_{a=1}^{N_{l}} \lambda_{a}(t) Z_{a}\left(\gamma_{\text {ref }}(t)\right)
$$

for $t \in I, N_{l} \in \mathbb{N}$, where $\lambda_{a}: I \rightarrow[0,+\infty)$ is of class $\mathcal{C}^{\infty}$.

We introduce for a curve on $Q$ the notion of having a regular parameterization on $\mathscr{K}_{i}$. A curve $\gamma: I \rightarrow Q$ admits a regular parameterization on $\mathscr{K}_{i}$ if there exist $N_{i} \in \mathbb{N}, \lambda_{1}, \ldots, \lambda_{N_{i}} \in \mathcal{C}(I)$ and $Z_{1}, \ldots, Z_{N_{i}} \in \mathscr{K}_{i}$ such that $\lambda_{a}(t) \geq 0$ for every $t \in I$ and $a \in\left\{1, \ldots, N_{i}\right\}$ and

$$
\nabla_{\dot{\gamma}(t)} \dot{\gamma}(t)-Y(t, \dot{\gamma}(t))=\sum_{a=1}^{N_{i}} \lambda_{a}(t) Z_{a}(\gamma(t)) .
$$

Fix $\epsilon>0$ and let $\xi_{l}=\gamma_{\text {ref. }}$ Then $\xi_{l}$ admits a regular parameterization on $\mathscr{K}_{l}$. We are going to prove by induction that there exists a finite sequence $\left\{\xi_{0}, \xi_{1}, \ldots, \xi_{l}\right\}$ of curves on $Q$ such that each $\xi_{i}$ satisfies $\dot{\xi}_{i}(0)=\dot{\gamma}_{\text {ref }}(0)$, admits a regular parameterization on $\mathscr{K}_{i}$ and $\mathrm{d}\left(\xi_{i}(t), \xi_{i+1}(t)\right)<\epsilon / l$ for every $i=0, \ldots, l-1$ and every $t \in I$. The induction step claims that: for $i=0, \ldots, l-1$ if there exists $\xi_{i+1}: I \rightarrow Q$ admitting a regular parameterization on $\mathscr{K}_{i+1}$, then there exists $\xi_{i}: I \rightarrow Q$ with $\dot{\xi}_{i+1}(0)=\dot{\xi}_{i}(0)$ admitting a regular parameterization on $\mathscr{K}_{i}$ and satisfying $\mathrm{d}\left(\xi_{i}(t), \xi_{i+1}(t)\right)<\epsilon / l$ for every $t \in I$.

Let us prove the induction step for $i$. Let $Z_{1}, \ldots, Z_{N_{i+1}} \in \mathscr{K}_{i+1}$ be the vector fields that determine the regular parameterization of $\xi_{i+1}$. Here we split the proof in two steps, considering first the special case:

1. $Z_{a}=F_{a}-G_{a}$ with $F_{a} \in \mathscr{K}_{i}$ and $G_{a} \in \operatorname{co}\left\{\langle Z: Z\rangle \mid Z \in \mathrm{L}\left(\mathscr{K}_{i}\right)\right\}$ for every $a=$ $1, \ldots, N_{i+1}$,

and then the general case

$$
\text { 2. } Z_{a} \in \overline{\mathscr{K}_{i}-\operatorname{co}\left\{\langle Z: Z\rangle \mid Z \in \mathrm{L}\left(\mathscr{K}_{i}\right)\right\}} \text {. }
$$

Let us study case 10: $\xi_{i+1}$ admits the parameterization

$$
\begin{array}{r}
\nabla_{\dot{\xi}_{i+1}(t)} \dot{\xi}_{i+1}(t)-Y\left(t, \dot{\xi}_{i+1}(t)\right)= \\
\sum_{a=1}^{N_{i+1}} \lambda_{a}(t)\left(F_{a}-G_{a}\right)\left(\xi_{i+1}(t)\right) .
\end{array}
$$


Each $G_{a}$ is given by $\sum_{b=1}^{N_{a}} \alpha_{a, b}\left\langle G_{a, b}: G_{a, b}\right\rangle$ where $G_{a, b} \in \mathrm{L}\left(\mathscr{K}_{i}\right)$ and $\alpha_{a, b} \geq 0$. Then (12) becomes

$$
\begin{gathered}
\nabla_{\dot{\xi}_{i+1}(t)} \dot{\xi}_{i+1}(t)-Y\left(t, \dot{\xi}_{i+1}(t)\right)=\sum_{a=1}^{N_{i+1}}\left(\lambda_{a}(t)\right. \\
\left.F_{a}\left(\xi_{i+1}(t)\right)-\sum_{b=1}^{N_{a}} \lambda_{a}(t) \alpha_{a, b}\left\langle G_{a, b}: G_{a, b}\right\rangle\left(\xi_{i+1}(t)\right)\right) .
\end{gathered}
$$

The dynamics described in (13) are of the same form as in (10) as long as we take $\mathscr{Y}=$ $\left\{G_{a, b}\right\}_{a=1, \ldots, N_{i+1} ; b=1, \ldots, N_{a}}$, and $\delta_{n_{a b} n_{a^{\prime} b^{\prime}}}=\delta_{a a^{\prime}} \delta_{b b^{\prime}}$, $w_{a, b}=\sqrt{\lambda_{a} \alpha_{a, b}}$.

Then by Proposition 4.2 the solutions to 13 ) can be approximated by solutions to

$$
\begin{gathered}
\nabla_{\dot{\xi}_{i} \epsilon_{i}(t)} \dot{\xi}_{i}^{\epsilon_{i}}(t)-Y\left(t, \dot{\xi}_{i}^{\epsilon_{i}}(t)\right)=\sum_{a=1}^{N_{i+1}}\left(\lambda_{a}(t) F_{a}\left(\xi_{i}^{\epsilon_{i}}(t)\right)\right. \\
\left.+\sum_{b=1}^{N_{a}} \frac{1}{\epsilon_{i}} \psi_{n_{a b}}\left(\frac{t}{\epsilon_{i}}\right) w_{a, b}(t) G_{a, b}\left(\xi_{i}^{\epsilon_{i}}(t)\right)\right)
\end{gathered}
$$

being $(a, b) \mapsto n_{a b}$ any injective map from $\mathbb{N} \times \mathbb{N}$ to $\mathbb{N}$ and $\left(\psi_{j}\right)_{j \in \mathbb{N}}$ a $\Lambda_{T}$-orthonormal and zero-mean sequence. More precisely, there exist $C_{i}, \epsilon_{i, 0}>0$ such that $\mathrm{d}\left(\xi_{i}^{\epsilon_{i}}(t), \xi_{i+1}(t)\right)<C_{i} \epsilon_{i}$ for every $t \in I$, $\epsilon_{i} \in\left(0, \epsilon_{i, 0}\right)$. In particular, we can choose $\epsilon_{i}$ such that $C_{i} \epsilon_{i}<\epsilon / l$.

The finite linear combination of elements in $\mathscr{K}_{i}\left(\xi_{i}^{\epsilon_{i}}(t)\right)$ for every $t \in I$ on the right-hand side of (14) does not necessarily satisfy the nonnegativeness of the coefficients. However, $G_{a, b} \in$ $\mathrm{L}\left(\mathscr{K}_{i}\right)$, so $-G_{a, b} \in \mathrm{L}\left(\mathscr{K}_{i}\right)$. Then we can rewrite the coefficients of $G_{a, b}$ as follows:

$$
\begin{gathered}
\psi_{n_{a b}}\left(\frac{t}{\epsilon_{i}}\right) w_{a, b}(t) G_{a, b}=\max \left\{0, \psi_{n_{a b}}\left(\frac{t}{\epsilon_{i}}\right)\right\} \\
w_{a, b}(t) G_{a, b}+\max \left\{0,-\psi_{n_{a b}}\left(\frac{t}{\epsilon_{i}}\right)\right\} w_{a, b}(t)\left(-G_{a, b}\right) .
\end{gathered}
$$

Thus all the coefficients are continuous and nonnegative. We can conclude that $\xi_{i}^{\epsilon_{i}}$ admits a regular parameterization on $\mathscr{K}_{i}$. We define then $\xi_{i}=\xi_{i}^{\epsilon_{i}}$ and the induction step has been proved for $i$ in the case 11.

Let us turn to case 2. We recall that the closure appearing in (9) is considered with respect to the topology of the uniform convergence on compact sets. Then there exist two sequences $F_{a}^{j} \in \mathscr{K}_{i}$ and $G_{a}^{j} \in \operatorname{co}\left\{\langle Z: Z\rangle \mid Z \in \mathrm{L}\left(\mathscr{K}_{i}\right)\right\}$ such that $F_{a}^{j}-G_{a}^{j}$ converges uniformly to $Z_{a}$ on a neighborhood of the curve $\xi_{i+1}$. For every $j \in \mathbb{N}$ let $\xi_{i+1}^{j}: I \rightarrow Q$ be the solution to

$$
\begin{aligned}
& \nabla_{\dot{\xi}_{i+1}^{j}(t)} \dot{\xi}_{i+1}^{j}(t)-Y\left(t, \dot{\xi}_{i+1}^{j}(t)\right) \\
& =\sum_{a=1}^{N_{i+1}} \lambda_{a}(t)\left(F_{a}^{j}-G_{a}^{j}\right)\left(\xi_{i+1}^{j}(t)\right)
\end{aligned}
$$

satisfying $\dot{\xi}_{i+1}^{j}(0)=\dot{\xi}_{i+1}(0)$. Then, thanks to Lemma 2.5, $\xi_{i+1}^{j}$ converges to $\xi_{i+1}$ uniformly on $I$ as $j$ tends to infinity. Take $\bar{\jmath}$ large enough such that

$$
\mathrm{d}\left(\xi_{i+1}^{\bar{\jmath}}(t), \xi_{i+1}(t)\right)<\frac{\epsilon}{2 l}
$$

for every $t \in I$. We can apply to $\xi_{i+1}^{\bar{j}}$ the same reasoning as in the first case. Then solutions to (15) are approximated by solutions to (14) replacing $\xi_{i}^{\epsilon_{i}}$ by $\xi_{i}^{\bar{\jmath}, \epsilon_{i}}, F_{a}$ by $F_{a}^{\bar{\jmath}}$ and $G_{a, b}$ by $G_{a, b}^{\bar{j}}$. In other words, there exist $C_{i}, \epsilon_{i, 0}>0$ such that $\mathrm{d}\left(\xi_{i}^{\bar{\jmath}, \epsilon_{i}}(t), \xi_{i+1}^{\bar{\jmath}}(t)\right)<$ $C_{i} \epsilon_{i}$ for every $t \in I, \epsilon_{i} \in\left(0, \epsilon_{i, 0}\right)$. Again, $\epsilon_{i}$ can be chosen in such a way that $C_{i} \epsilon_{i}<\frac{\epsilon}{2 l}$ and we define $\xi_{i}=\xi_{i}^{\bar{\jmath}, \epsilon_{i}}$. Thus,

$$
\begin{aligned}
\mathrm{d}\left(\xi_{i}(t), \xi_{i+1}(t)\right) & \leq \mathrm{d}\left(\xi_{i}^{\bar{\jmath}, \epsilon_{i}}(t), \xi_{i+1}^{\bar{\jmath}}(t)\right) \\
& +\mathrm{d}\left(\xi_{i+1}^{\bar{j}}(t), \xi_{i+1}(t)\right)<\frac{\epsilon}{l}
\end{aligned}
$$

and $\xi_{i}$ admits a regular parameterization on $\mathscr{K}_{i}$. Hence, the induction step has been proved for $i$.

After the induction, we end up with a curve $\xi_{0}$ on $Q$ admitting a regular parameterization on $\mathscr{K}_{0}$ and such that

$$
\mathrm{d}\left(\xi_{0}(t), \gamma_{\mathrm{ref}}(t)\right) \leq \sum_{i=0}^{l-1} \mathrm{~d}\left(\xi_{i}(t), \xi_{i+1}(t)\right)<l \frac{\epsilon}{l}=\epsilon
$$

for every $t \in I$. (Recall that $\xi_{l}=\gamma_{\text {ref }}$.) Moreover, by compactness of $I$,

$$
\bar{\epsilon}=\epsilon-\max _{t \in I} \mathrm{~d}\left(\xi_{0}(t), \gamma_{\mathrm{ref}}(t)\right)>0 .
$$

As $\mathscr{K}_{0}=\overline{\operatorname{span}_{\mathcal{C}^{\infty}(Q)} \mathscr{Y}}$, we conclude from Lemma 2.5 that there exist $\theta_{a, j} \in \mathcal{C}^{\infty}(Q), a \in\left\{1, \ldots, N_{0}\right\}, j \in$ $\{1, \ldots, k\}$, such that the solution to

$$
\begin{aligned}
\nabla_{\dot{\xi}(t)} \dot{\xi}(t) & -Y(t, \dot{\xi}(t)) \\
& =\sum_{a=1}^{N_{0}} \sum_{j=1}^{k} \lambda_{a}(t) \theta_{a, j}(\xi(t)) Y_{j}(\xi(t))
\end{aligned}
$$

with initial condition $\dot{\xi}(0)=\dot{\xi}_{0}(0)=\dot{\gamma}_{\text {ref }}(0)$ satisfies $\mathrm{d}\left(\xi(t), \xi_{0}(t)\right)<\bar{\epsilon}$ for every $t \in I$. Thus, $\mathrm{d}\left(\xi(t), \gamma_{\text {ref }}(t)\right)<\epsilon$ for every $t \in I$.

Since $\xi$ is an admissible trajectory for $\Sigma$, we conclude that $\gamma_{\text {ref }}$ is trackable for $\Sigma$ with the tracking control law given by $u_{j}(t)=\sum_{a=1}^{N_{0}} \lambda_{a}(t) \theta_{a, j}(\xi(t))$, $j=1, \ldots, k$. 
Corollary 4.5. Let $\Sigma=\left(Q, \nabla, Y, \mathscr{Y}, \mathbb{R}^{k}\right)$ be a FACCS. Define the following set of vector fields on $Q$ for $l \in \mathbb{N}$ :

$$
\begin{aligned}
& \mathscr{H}_{0}=\operatorname{span}_{\mathcal{C}^{\infty}(Q)} \mathscr{Y} \\
& \mathscr{H}_{l}=\mathscr{H}_{l-1}-\operatorname{co}\left\{\langle Z: Z\rangle \mid Z \in \mathrm{L}\left(\mathscr{H}_{l-1}\right)\right\}
\end{aligned}
$$

Fix a smooth reference trajectory $\gamma_{\mathrm{ref}}: I \rightarrow Q$. If for every $t \in I$ there exists $l \in \mathbb{N}$ such that $\mathscr{H}_{l}\left(\gamma_{\text {ref }}(t)\right)=$ $T_{\gamma_{\mathrm{ref}}(t)} Q$, then $\gamma_{\mathrm{ref}}$ is trackable. Therefore, if for every $q \in Q$ there exists $l \in \mathbb{N}$ such that $\mathscr{H}_{l}(q)=$ $T_{q} Q$, then the control system $\Sigma$ is trackable.

Proof. Let us prove by induction that

$$
\mathscr{H}_{i} \subseteq \mathscr{K}_{i} \quad i \in \mathbb{N}_{0} .
$$

It is trivial that $\mathscr{H}_{0} \subseteq \mathscr{K}_{0}$ (see (9) and (16)).

The claim now is that if $\mathscr{H}_{i} \subseteq \mathscr{K}_{i}$, then $\mathscr{H}_{i+1} \subseteq \mathscr{K}_{i+1}$. By definition, an element in $\mathscr{H}_{i+1}$ is of the form $F-G$ with $F \in \mathscr{H}_{i}$ and $G \in \operatorname{co}\left\{\langle Z: Z\rangle \mid Z \in \mathrm{L}\left(\mathscr{H}_{i}\right)\right\}$. Since $\mathscr{H}_{i} \subseteq \mathscr{K}_{i}$, then $F \in \mathscr{K}_{i}$ and $\mathrm{L}\left(\mathscr{H}_{i}\right) \subseteq \mathrm{L}\left(\mathscr{K}_{i}\right)$. So $G \in$ co $\left\{\langle Z: Z\rangle \mid Z \in \mathrm{L}\left(\mathscr{K}_{i}\right)\right\}$. We can conclude that $\mathscr{H}_{i+1} \subseteq \mathscr{K}_{i+1}$.

By hypotheses, for every $t \in I$ there exists $l \in$ $\mathbb{N}$ such that $\mathscr{H}_{l}\left(\gamma_{\mathrm{ref}}(t)\right)=T_{\gamma_{\mathrm{ref}}(t)} Q$. As $\mathscr{H}_{l} \subseteq \mathscr{K}_{l}$, we have $\mathscr{K}_{l}\left(\gamma_{\text {ref }}(t)\right)=T_{\gamma_{\text {ref }}(t)} Q$. The hypotheses of Theorem 4.4 are satisfied, so the result holds.

Remark 4.6. If one follows the proof of Theorem 4.4 under the stronger hypotheses of Corollary 4.5, then each step of the induction procedure is of the type considered in case 1. This will be important in the next section, where we will turn such procedure in an algorithmic construction.

Corollary 4.7. Let $\Sigma=\left(Q, \nabla, Y, \mathscr{Y}, \mathbb{R}^{k}\right)$ be a FACCS. Define the following sets of vector fields for $l \in \mathbb{N}$,

$$
\begin{aligned}
\mathscr{Z}_{0} & =\mathscr{Y}, \\
\mathscr{Z}_{l} & =\mathscr{Z}_{l-1} \cup\left\{\left\langle Z_{a}: Z_{b}\right\rangle \mid Z_{a}, Z_{b} \in \mathscr{Z}_{l-1}\right\} .
\end{aligned}
$$

If there exists $l \in \mathbb{N}$ such that $\operatorname{span}_{\mathbb{R}} \mathscr{Z}_{l}(q)=T_{q} Q$ for all $q \in Q$ and for each $i \in\{0, \ldots, l-1\}$, for each $Z \in \mathscr{Z}_{i},\langle Z: Z\rangle \in \operatorname{span}_{\mathcal{C}^{\infty}(Q)} \mathscr{Z}_{i}$, then the system $\Sigma$ is trackable.

Proof. Let us prove by induction that

$$
\operatorname{span}_{\mathcal{C}^{\infty}(Q)} \mathscr{Z}_{i} \subseteq \mathscr{K}_{i}, \quad i \in \mathbb{N}_{0}
$$

Once this inclusion is proved Theorem 4.4 guarantees the trackability of the system.
It is trivial by definition that $\operatorname{span}_{\mathcal{C}^{\infty}(Q)} \mathscr{Z}_{0} \subseteq \mathscr{K}_{0}$.

Assume that $\operatorname{span}_{\mathcal{C} \infty(Q)} \mathscr{Z}_{i} \subseteq \mathscr{K}_{i}$ and let us prove the inclusion for $i$. Since $\mathscr{K}_{i+1}$ is a convex cone by Proposition 4.1 and $\operatorname{span}_{\mathcal{C}^{\infty}(Q)} \mathscr{Z}_{i} \subset \mathscr{K}_{i+1}$, it is enough to prove that $\alpha\left\langle Z_{a}: Z_{b}\right\rangle$ belongs to $\mathscr{K}_{i+1}$ for $Z_{a}, Z_{b} \in \mathscr{Z}_{i}$ and $\alpha \in \mathcal{C}^{\infty}(Q)$. Thanks to (ब1) and to the hypotheses on the symmetric products of elements of $\mathscr{Z}_{i}$,

$$
\begin{array}{r}
\left\langle\alpha Z_{a}: Z_{b}\right\rangle \in \alpha\left\langle Z_{a}: Z_{b}\right\rangle+\operatorname{span}_{\mathcal{C}^{\infty}(Q)} \mathscr{Z}_{i}, \\
\left\langle\alpha Z_{a}: \alpha Z_{a}\right\rangle,\left\langle Z_{b}: Z_{b}\right\rangle \in \operatorname{span}_{\mathcal{C}^{\infty}(Q)} \mathscr{Z}_{i} .
\end{array}
$$

Hence the symmetric product

$$
\left\langle\alpha Z_{a}-Z_{b}: \alpha Z_{a}-Z_{b}\right\rangle
$$

belongs to $-2 \alpha\left\langle Z_{a}: Z_{b}\right\rangle+\operatorname{span}_{\mathcal{C}^{\infty}(Q)} \mathscr{Z}_{i}$. Thus, $\alpha\left\langle Z_{a}: Z_{b}\right\rangle$ belongs to $\operatorname{span}_{\mathcal{C}^{\infty}(Q)} \mathscr{Z}_{i}-\frac{1}{2}\left\langle\alpha Z_{a}-\right.$ $\left.Z_{b}: \alpha Z_{a}-Z_{b}\right\rangle$, which is contained in $\mathscr{K}_{i+1}$.

Remark 4.8. The proof above actually shows that, under the assumptions of Corollary 4.7, $\operatorname{span}_{\mathcal{C}^{\infty}(Q)} \mathscr{Z}_{i} \subseteq \mathscr{H}_{i}$. It is easy to check that, in addition, $\mathscr{H}_{i}=\operatorname{span}_{\mathcal{C}^{\infty}(Q)} \mathscr{Z}_{i}$.

Remark 4.9. The main interest of Corollary 4.7 is that its hypotheses are formulated in terms of a finite set of vector fields, in contrast with the infinite family of vector fields considered in Theorem 4.4 and Corollary 4.5 .

\subsection{Examples}

Let us consider some examples of mechanical systems for which the above results guarantee the trackability, but Theorem 3.3 could not guarantee it.

\subsubsection{Hovercraft}

Consider an elliptic hovercraft moving on the surface of a fluid, identified with $\mathbb{R}^{2}$. The configuration manifold is $Q=S^{1} \times \mathbb{R}^{2}$ with local coordinates $\left(\theta, x_{1}, x_{2}\right)$ where $\theta$ is the attitude and $\left(x_{1}, x_{2}\right)$ is the position of the center of symmetry of the hovercraft. Let $\omega$ and $\left(v_{1}, v_{2}\right)$ be the standard angular and linear velocity, respectively, of the hovercraft with respect to a body-fixed coordinate frame attached at the center of symmetry of the body and whose axes coincide with those of the ellipse. Assume that the center of mass according to that body-fixed coordinate frame is on the horizontal axis and is different 
from the center of symmetry. Then the added inertia matrix is the following $3 \times 3$ symmetric matrix:

$$
\mathcal{M}=\left(\begin{array}{l|ll}
a & 0 & c \\
\hline 0 & e & 0 \\
c & 0 & e
\end{array}\right),
$$

with $a, c, e>0$ (see 9, 13] for more details). Denote the corresponding impulse vector by $\left(\Pi, P_{1}, P_{2}\right)$ that is related to the velocities through the inertia matrix $\mathcal{M}$ as follows

$$
\left(\begin{array}{l}
\Pi \\
P_{1} \\
P_{2}
\end{array}\right)=\mathcal{M}\left(\begin{array}{l}
\omega \\
v_{1} \\
v_{2}
\end{array}\right)
$$

The dynamics of the systems governed by the Kirchhoff equations in dimension 2 with two controls are

$$
\begin{aligned}
\left(\begin{array}{c}
\dot{\theta} \\
\dot{x} \\
\dot{y}
\end{array}\right) & =\left(\begin{array}{ccc}
1 & 0 & 0 \\
0 & \cos \theta & -\sin \theta \\
0 & \sin \theta & \cos \theta
\end{array}\right)\left(\begin{array}{c}
\omega \\
x \\
y
\end{array}\right), \\
\left(\begin{array}{c}
\dot{\omega} \\
\dot{v}_{1} \\
\dot{v}_{2}
\end{array}\right) & =\mathcal{M}^{-1}\left(\begin{array}{c}
P \cdot v^{\perp} \\
\omega P^{\perp}
\end{array}\right)+\left(\begin{array}{c}
u_{1} \\
0 \\
u_{2}
\end{array}\right)
\end{aligned}
$$

where $w^{\perp}=\left(-w_{2}, w_{1}\right)$ denotes the rotation by $\pi / 2$ of a vector $w=\left(w_{1}, w_{2}\right)$ in $\mathbb{R}^{2}$. The control vector fields are $Y_{1}=(1,0,0)$ and $Y_{2}=(0,0,1)$. They correspond to the external torque, usually called yaw, and the external force, usually called surge, applied to the body. Let $\mathscr{Y}=\left\{Y_{1}, Y_{2}\right\}$.

The drift term $Z$ appearing in (3) is, for the mechaincal system considered here, the vector field corresponding to the uncontrolled Kirchhoff's equations (notice that $Y=0$ ). Using (4) we can compute $\left\langle Y_{1}: Y_{1}\right\rangle=(0,2 c / e, 0)$. The sufficient conditions for tracking given by Theorem 3.3 are not satisfied because $\left\langle Y_{1}: Y_{1}\right\rangle \notin \operatorname{span}_{\mathcal{C}^{\infty}(Q)}\left\{Y_{1}, Y_{2}\right\}$.

However, due to Corollary 1.5 tracking is possible because

$$
\mathscr{H}_{1}(q)=T_{q} Q \forall q \in Q .
$$

Indeed, for $w_{1}, w_{2} \in \mathcal{C}^{\infty}(Q)$,

$$
\begin{aligned}
& \left\langle w_{1} Y_{1}+w_{2} Y_{2}: w_{1} Y_{1}+w_{2} Y_{2}\right\rangle= \\
& \frac{1}{e}\left(0,2 c w_{1}^{2}+2 e w_{1} w_{2}, 0\right)+2 \overbrace{\sum_{i, j=1}^{2} w_{i} Y_{i}\left(w_{j}\right) Y_{j}}^{\in \operatorname{span}_{\mathcal{C} \infty(Q)} \mathscr{Y}},
\end{aligned}
$$

where $Y_{i}\left(w_{j}\right)$ denotes the Lie derivative of $w_{j}$ with respect to $Y_{i}$. In particular, taking as $w_{1}$ any nonzero constant function, we get

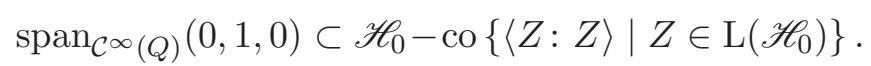

So we conclude that $\mathscr{H}_{1}(q)=T_{q} Q$ for all $q \in Q$.

\subsubsection{Submarine}

Let us apply Corollary 4.7 to determine the trackability of a particular control system describing the motion of a submarine. The system corresponds to the case $\gamma=0$ considered in [6]. It models a neutrally buoyant ellipsoid vehicle immersed in a infinite volume fluid that is inviscid, incompressible and whose motion is irrotational. The dynamics are obtained through Kirchhoff equations [9] and have a particularly simple form due to some symmetry assumption on the distribution of mass (see [6] for details and also [14] for general overview of control motion in a potential fluid).

Consider the coordinates $(\omega, v)$ for the angular and linear velocity of the ellipsoid with respect to a body-fixed coordinate frame. Then the impulse $(\Pi, P)$ of the system is given by

$$
\left(\begin{array}{l}
\Pi \\
P
\end{array}\right)=\mathcal{M}\left(\begin{array}{l}
\omega \\
v
\end{array}\right)
$$

where, under the symmetry assumptions mentioned above,

$$
\mathcal{M}=\operatorname{diag}\left(J_{1}, J_{1}, J_{3}, M_{1}, M_{2}, M_{3}\right)
$$

with $M_{1} \neq M_{2}$, where $\operatorname{diag}\left(J_{1}, J_{1}, J_{3}\right)$ is the usual inertia matrix and $M_{1}, M_{2}, M_{3}$ take into account the mass of the submarine and the added masses due to the action of the fluid.

The configuration manifold $Q$ for this problem is the Special Euclidean group or the group of rigid motions $S E(3)$, which is homeomorphic to $\mathbb{R}^{3} \times S O(3)$. Let $(r, A) \in S E(3)$ be the position and the attitude of the ellipsoid. Denote by $S: \mathbb{R}^{3} \rightarrow \mathfrak{s o}(3)$ the linear bijection between $\mathbb{R}^{3}$ and the linear algebra $\mathfrak{s o}(3)$ of $S O(3)$ such that

$$
S\left(x_{1}, x_{2}, x_{3}\right)=\left(\begin{array}{ccc}
0 & -x_{3} & x_{2} \\
x_{3} & 0 & -x_{1} \\
-x_{2} & x_{1} & 0
\end{array}\right)
$$

The dynamics of the controlled system are given by

$$
\frac{\mathrm{d} r}{\mathrm{~d} t}=A v, \quad \frac{\mathrm{d} A}{\mathrm{~d} t}=A S(\omega)
$$

and

$$
\frac{\mathrm{d} \Pi}{\mathrm{d} t}=\Pi \times \omega+P \times v+\left(\begin{array}{c}
u_{1} \\
u_{2} \\
0
\end{array}\right), \quad \frac{\mathrm{d} P}{\mathrm{~d} t}=P \times \omega+\left(\begin{array}{c}
0 \\
0 \\
u_{3}
\end{array}\right) .
$$

The control vector fields are $Y_{1}=\partial / \partial \Pi_{1}, Y_{2}=$ $\partial / \partial \Pi_{2}$ and $Y_{3}=\partial / \partial P_{3}$. They correspond to a linear acceleration along one of the three axes of the 
submarine and to two angular accelerations around the other two axes.

Due to Theorem 1.2 in [6], we know that system (18)-(19) is trackable.

This case cannot be recovered from Theorem 3.3 because

$$
\operatorname{Sym}^{(1)}(\mathscr{Y}) \neq T Q .
$$

Indeed, it can be computed that

$$
\begin{aligned}
& \left\langle Y_{1}: Y_{2}\right\rangle=0,\left\langle Y_{2}: Y_{3}\right\rangle=\frac{M_{3}}{M_{1}} \frac{\partial}{\partial P_{1}}, \\
& \left\langle Y_{1}: Y_{3}\right\rangle=-\frac{M_{3}}{M_{2}} \frac{\partial}{\partial P_{2}} .
\end{aligned}
$$

However, this case is covered by Corollary 4.7 with $l=2$, because

$$
\begin{aligned}
& 0=\left\langle Y_{1}: Y_{1}\right\rangle=\left\langle Y_{2}: Y_{2}\right\rangle=\left\langle Y_{3}: Y_{3}\right\rangle= \\
& =\left\langle\left\langle Y_{2}: Y_{3}\right\rangle:\left\langle Y_{2}: Y_{3}\right\rangle\right\rangle=\left\langle\left\langle Y_{1}: Y_{3}\right\rangle:\left\langle Y_{1}: Y_{3}\right\rangle\right\rangle \\
& \left\langle\left\langle Y_{2}: Y_{3}\right\rangle:\left\langle Y_{1}: Y_{3}\right\rangle\right\rangle=-\frac{M_{3}^{2}}{J_{3}}\left(\frac{1}{M_{1}}-\frac{1}{M_{2}}\right) \frac{\partial}{\partial \Pi_{3}} .
\end{aligned}
$$

Thus, $\langle Z: Z\rangle=0$ for every $Z \in$ $\mathscr{Z}_{2}$ and $T_{q} Q=\operatorname{span}_{\mathbb{R}} \mathscr{Z}_{2}(q)=$ $\operatorname{span}_{\mathbb{R}}\left\{Y_{1}(q), Y_{2}(q), Y_{3}(q),\left\langle Y_{2}: Y_{3}\right\rangle(q),\left\langle Y_{1}: Y_{3}\right\rangle(q)\right.$, $\left.\left\langle\left\langle Y_{2}: Y_{3}\right\rangle:\left\langle Y_{1}: Y_{3}\right\rangle\right\rangle(q)\right\}$ for all $q \in Q$.

The model studied in [6] can therefore be handled with the techniques proposed here. In particular, we can obtain for it one-parameter tracking control laws, as explained in the next section.

\section{One-parameter tracking con- trol laws}

The aim of this section is to provide an algorithmic implementation of the results obtained in the previous one about the existence of controls yielding tracking. This will be done separately under the hypotheses of Corollaries 4.5 and 4.7, using two different algorithms. The first one is based on the procedure proposed in the proof of Theorem 4.4, while the second one exploits the construction proposed in [5] and recalled in Theorem 3.3.

In both cases we will consider a reference trajectory $\gamma_{\text {ref }}: I \rightarrow Q$, which is assumed to be of class $\mathcal{C}^{\infty}$.

A simple, albeit crucial, fact that will be used several times in the following sections is stated in the lemma below.

Lemma 5.1. If $f: \mathbb{R} \times I \rightarrow \mathbb{R},(\tau, s) \mapsto f(\tau, s)$, is smooth on $\mathbb{R} \times I$ and $T$-periodic with respect to $\tau$, then

$$
\begin{aligned}
\int_{0}^{t} f(s / \hat{\epsilon}, s) \mathrm{d} s & =\int_{0}^{t} \bar{f}(s) d s+O\left(\hat{\epsilon}\|f\|_{\infty}\right) \\
& +O\left(\hat{\epsilon}\left\|\partial_{2} f\right\|_{\infty}\right)
\end{aligned}
$$

for $\hat{\epsilon}$ close to zero, where $\bar{f}(s)=(1 / T) \int_{0}^{T} f(\tau, s) \mathrm{d} \tau$ and $\partial_{2}$ denotes the partial derivative with respect to the second variable.

\subsection{Case $\mathscr{H}$}

As noticed in Remark 4.6, the hypotheses of Corollary 4.5 guarantee that every step of the induction argument proposed in the proof of Theorem 1.4 falls in the framework of case 1 (see page 6). Hence, starting from a parameterization

$$
\nabla_{\dot{\gamma}_{\text {ref }}(t)} \dot{\gamma}_{\text {ref }}(t)-Y\left(t, \dot{\gamma}_{\text {ref }}(t)\right)=\sum_{a=1}^{N_{l}} \lambda_{a}(t) Z_{a}^{l}\left(\gamma_{\text {ref }}(t)\right)
$$

of $\gamma_{\mathrm{ref}}: I \rightarrow Q$, with $Z_{a}^{l} \in \mathscr{H}_{l}$ and $\lambda_{a}$ smooth and non-negative on $I$ for every $a=1, \ldots, N_{l}$, we can construct algorithmically a $l$-parameter family of admissible trajectories $\xi^{\epsilon_{1}, \ldots, \epsilon_{l}}$ of $\Sigma$ with $\epsilon_{1}, \ldots, \epsilon_{l}>$ 0 such that $\xi_{l}=\gamma_{\text {ref }}$, the uniform limit

$$
\xi_{i}^{\epsilon_{1}, \ldots, \epsilon_{l-i}}=\lim _{\epsilon_{l} \rightarrow 0} \lim _{\epsilon_{l-1} \rightarrow 0} \cdots \lim _{\epsilon_{l-i+1} \rightarrow 0} \xi^{\epsilon_{1}, \ldots, \epsilon_{l}}
$$

exists for every $i=1, \ldots, l$ and every $\epsilon_{1}, \ldots, \epsilon_{l-i}>0$ and satisfies

$$
\begin{aligned}
\nabla_{\dot{\xi}_{i}^{\epsilon_{1}}, \ldots, \epsilon_{l-i}(t)} \dot{\xi}_{i}^{\epsilon_{1}, \ldots, \epsilon_{l-i}}(t) & -Y\left(t, \dot{\xi}_{i}^{\epsilon_{1}, \ldots, \epsilon_{l-i}}(t)\right) \\
& \in \mathscr{H}_{i}\left(\xi_{i}^{\epsilon_{1}, \ldots, \epsilon_{l-i}}(t)\right) .
\end{aligned}
$$

We also write $\xi_{0}^{\epsilon_{1}, \ldots, \epsilon_{l}}$ for $\xi^{\epsilon_{1}, \ldots, \epsilon_{l}}$. It is important to notice that the order of the limits in (20) cannot in general be reversed.

Let us recall to which extent the construction is algorithmic. Fix any injective map $j: \mathbb{N} \times \mathbb{N} \rightarrow \mathbb{N}$. By backward recursion on $i$, if $\xi_{i}^{\epsilon_{1}, \ldots, \epsilon_{l-i}}$ satisfies

$$
\begin{array}{r}
\nabla_{\dot{\xi}_{i}^{\epsilon_{1}, \ldots, \epsilon_{l-i}}(t)} \dot{\xi}_{i}^{\epsilon_{1}, \ldots, \epsilon_{l-i}}(t)-Y\left(t, \dot{\xi}_{i}^{\epsilon_{1}, \ldots, \epsilon_{l-i}}(t)\right) \\
=\sum_{a=1}^{N_{i}} \lambda_{a}^{\epsilon_{1}, \ldots, \epsilon_{l-i}}(t) Z_{a}^{i}\left(\xi_{i}^{\epsilon_{1}, \ldots, \epsilon_{l-i}}(t)\right)
\end{array}
$$

with $\lambda_{a}^{\epsilon_{1}, \ldots, \epsilon_{l-i}} \in \mathcal{C}(I,[0,+\infty))$ and $Z_{a}^{i} \in \mathscr{H}_{i}$, then 
$\xi_{i-1}^{\epsilon_{1}, \ldots, \epsilon_{l-i+1}}$ is defined as the solution to

$$
\begin{aligned}
& \nabla_{\dot{\xi}_{i-1}^{\epsilon_{1}, \ldots, \epsilon_{l-i+1}}(t)} \dot{\xi}_{i-1}^{\epsilon_{1}, \ldots, \epsilon_{l-i+1}}(t) \\
& -Y\left(t, \dot{\xi}_{i-1}^{\epsilon_{1}, \ldots, \epsilon_{l-i+1}}(t)\right) \\
& =\sum_{a=1}^{N_{i}}\left(\lambda_{a}^{\epsilon_{1}, \ldots, \epsilon_{l-i}}(t) F_{a}^{i-1}\left(\xi_{i-1}^{\epsilon_{1}, \ldots, \epsilon_{l-i+1}}(t)\right)+\right. \\
& \frac{1}{\epsilon_{l-i+1}} \sum_{b=1}^{\hat{N}_{a}^{i}} \psi_{j(a, b)}\left(\frac{t}{\epsilon_{l-i+1}}\right) \sqrt{\lambda_{a}^{\epsilon_{1}, \ldots, \epsilon_{l-i}}(t) \alpha_{a, b}^{i-1}} \\
& \left.G_{a, b}^{i-1}\left(\xi_{i-1}^{\epsilon_{1}, \ldots, \epsilon_{l-i+1}}(t)\right)\right)
\end{aligned}
$$

with $\dot{\xi}_{i-1}^{\epsilon_{1}, \ldots, \epsilon_{l-i+1}}(0)=\dot{\gamma}_{\text {ref }}(0)$, where

$$
Z_{a}^{i}=F_{a}^{i-1}-\sum_{b=1}^{\hat{N}_{a}^{i}} \alpha_{a, b}^{i-1}\left\langle G_{a, b}^{i-1}: G_{a, b}^{i-1}\right\rangle
$$

and $F_{a}^{i-1} \in \mathscr{H}_{i-1}, G_{a, b}^{i-1} \in \mathrm{L}\left(\mathscr{H}_{i-1}\right), \alpha_{a, b}^{i-1} \geq 0$. Recall that $\left(\psi_{j}\right)_{j \in \mathbb{N}}$ is a $\Lambda_{T}$-orthonormal and zeromean sequence, for some $T>0$. Each $\lambda_{a}^{\epsilon_{1}, \ldots, \epsilon_{l-i+1}}(\cdot)$ is either equal to some $\lambda_{\tilde{a}}^{\epsilon_{1}, \ldots, \epsilon_{l-i}}(\cdot)$ or is of the form

$$
\frac{\sqrt{\lambda_{\tilde{a}}^{\epsilon_{1}, \ldots, \epsilon_{l-i}}(\cdot) \alpha_{\tilde{a}, b}^{i-1}}}{\epsilon_{l-i+1}} \max \left\{v \psi_{j(\tilde{a}, b)}\left(\frac{\cdot}{\epsilon_{l-i+1}}\right), 0\right\}
$$

with $v$ equal to 1 or -1 .

We choose $\left(\psi_{j}\right)_{j \in \mathbb{N}}$ as follows: we require $\psi_{1}$ to be positive in $(0, T / 2)$ and to annihilate, together with all its derivatives, at 0 and $T / 2$. We also require it to satisfy

$$
\psi_{1}\left(\frac{T}{2}-t\right)=\psi_{1}(t), \quad t \in\left[0, \frac{T}{2}\right]
$$

and we extend it by

$$
\psi_{1}(t)=-\psi_{1}\left(t-\frac{T}{2}\right), \quad t \in\left[\frac{T}{2}, T\right],
$$

and by $T$-periodicity over $\mathbb{R}$. Finally we normalize $\psi_{1}$ in such a way that $\Lambda_{T}\left(\psi_{1}, \psi_{1}\right)=1$. Then we define $\psi_{j}$ by

$$
\psi_{j}(t)=2^{j} \psi_{1}\left(2^{j} t\right)
$$

Such choice of $\left(\psi_{j}\right)_{j \in \mathbb{N}}$ is motivated by the property that, for every choice of $j \in \mathbb{N}, l \in \mathbb{N}_{0}$ and $v \in\{-1,1\}$, the function $t \mapsto \sqrt[2^{l}]{\max \left\{v \psi_{j}(t), 0\right\}}$ is smooth. In particular, by backward recursion, each $\lambda_{a}^{\epsilon_{1}, \ldots, \epsilon_{i}}$ is smooth and is the product of functions of the type $\sqrt[2^{l}]{\frac{1}{\epsilon_{h}} \max \left\{v \psi_{j}\left(\frac{\dot{\epsilon_{h}}}{)}, 0\right\}\right.}$ and of $\sqrt[2^{m}]{\lambda_{b}}$ for some $m \in \mathbb{N}_{0}$ and some $b \in\left\{1, \ldots, N_{l}\right\}$.
An important consequence of this factorization, which will be exploited in the proof of Theorem 5.2, is that the derivatives of $\sqrt{\lambda_{a}^{\epsilon_{1}, \ldots, \epsilon_{i}}}$ with respect to time can be bounded by a finite constant depending explicitly on $\epsilon_{1}, \ldots, \epsilon_{l-1}$.

The smoothness of $\lambda_{a}^{\epsilon_{1}, \ldots, \epsilon_{i}}$, moreover, allows us to consider $\lambda_{a}^{\epsilon_{1}, \ldots, \epsilon_{i}} Z_{a}^{l-i}$ as a smooth vector field on the extended manifold $\mathbb{R} \times Q$ and, similarly, $\lambda_{a}^{\epsilon_{1}, \ldots, \epsilon_{l-i}}\left(Z_{a}^{i}\right)^{V}$ as a smooth vector field on the extended manifold $\mathbb{R} \times T Q$.

Summing up, the trajectories of the $l$-parameter family $\xi_{0}^{\epsilon_{1}, \ldots, \epsilon_{l}}=\xi^{\epsilon_{1}, \ldots, \epsilon_{l}}$ are driven by a $l$-parameter family of control laws $u^{\epsilon_{1}, \ldots, \epsilon_{l}} \in \mathcal{C}^{\infty}\left(I, \mathbb{R}^{k}\right)$ depending smoothly on $\epsilon_{1}, \ldots, \epsilon_{l}$. The construction of Theorem 4.4 can be summarized as follows: given $\epsilon>0$, if

$$
0<\epsilon_{l} \ll \epsilon_{l-1} \ll \cdots \ll \epsilon_{1} \ll 1
$$

then $\mathrm{d}\left(\xi^{\epsilon_{1}, \ldots, \epsilon_{l}}(t), \gamma_{\text {ref }}(t)\right)<\epsilon$ for every $t \in I$. Our aim is here to quantify the relations in (24). More precisely, we introduce $l-1$ functions $\eta_{2}, \ldots, \eta_{l}$ : $(0,+\infty) \rightarrow(0,+\infty)$ and we look for asymptotic conditions on their convergence to zero at zero such that

$$
\lim _{\epsilon \rightarrow 0} \mathrm{~d}\left(\xi^{\epsilon, \eta_{2}(\epsilon), \eta_{3} \circ \eta_{2}(\epsilon), \ldots, \eta_{l} \circ \cdots \circ \eta_{2}(\epsilon)}(t), \gamma_{\mathrm{ref}}(t)\right)=0
$$

uniformly with respect to $t \in I$. Let $\hat{\eta}_{i}=\eta_{i}$ 。 $\cdots \circ \eta_{2}$ for $i=2, \ldots, l$ and define $\hat{\eta}_{1}$ as the identity on $(0,+\infty)$. We say that $\epsilon \mapsto u^{\hat{\eta}_{1}(\epsilon), \ldots, \hat{\eta}_{l}(\epsilon)}$ is a one-parameter tracking control law for $\gamma_{\mathrm{ref}}$ if (25) holds true.

Theorem 5.2. Let $\Sigma=\left(Q, \nabla, Y, \mathscr{Y}, \mathbb{R}^{k}\right)$ be a FACCS. Let $\mathscr{H}_{i}, i \in \mathbb{N}_{0}$, be defined as in (16). Fix a reference trajectory $\gamma_{\mathrm{ref}} \in \mathcal{C}^{\infty}(I, Q)$ and assume that there exists $l \in \mathbb{N}$ such that

$$
\nabla_{\dot{\gamma}_{\text {ref }}(t)} \dot{\gamma}_{\text {ref }}(t)-Y\left(t, \dot{\gamma}_{\text {ref }}(t)\right) \in \mathscr{H}_{l}\left(\gamma_{\text {ref }}(t)\right)
$$

for every $t \in I$. Construct $\xi^{\epsilon_{1}, \ldots, \epsilon_{l}}, u^{\epsilon_{1}, \ldots, \epsilon_{l}}$ and $\hat{\eta}_{i}$ as above. If $\eta_{i}:(0,+\infty) \rightarrow(0,+\infty)$ satisfies $\lim \sup _{\epsilon \rightarrow 0} \eta_{i}(\epsilon) / \epsilon^{3}<\infty$ for every $i=2, \ldots, l$, then $\epsilon \mapsto u^{\hat{\eta}_{1}(\epsilon), \ldots, \hat{\eta}_{l}(\epsilon)}$ is a one-parameter tracking control law for $\gamma_{\mathrm{ref}}$.

Proof. The first step of the proof consists in estimating the order with respect to $\epsilon$ of the $L^{\infty}$-norm of the time-dependent parameters appearing in the parameterization (22). We write $\epsilon_{i}$ for $\hat{\eta}_{i}(\epsilon)$. Denoting by $C$ any constant not depending on the $\epsilon_{j}$, it is easy to check by backward induction on $i=0, \ldots, l$ that

$$
\left\|\lambda_{a}^{\epsilon_{1}, \ldots, \epsilon_{i}}\right\|_{\infty} \leq \frac{C}{\sqrt[2^{i-1}]{\epsilon_{1}} \sqrt[2^{i-2}]{\epsilon_{2}} \cdots \sqrt{\epsilon_{i-1}} \epsilon_{i}} .
$$


Exploiting the factorization of $\lambda_{a}^{\epsilon_{1}, \ldots, \epsilon_{i}}$ described above we get, in addition,

$$
\left\|\frac{\mathrm{d}^{m}}{\mathrm{~d} t^{m}} \sqrt{\lambda_{a}^{\epsilon_{1}, \ldots, \epsilon_{i}}}\right\|_{\infty} \leq \frac{C}{\sqrt[2^{i}]{\epsilon_{1}} 2^{i-1} \sqrt{\epsilon_{2}} \cdots \sqrt[4]{\epsilon_{i-1}} \epsilon_{i}^{m+\frac{1}{2}}}
$$

for $m \in \mathbb{N}_{0}$.

Consider the extended system on $\mathbb{R} \times T Q$ associated with (3) where the time is the new variable. In the following computations we write, using the notation introduced in (23),

$$
\begin{aligned}
X^{e} & =\left(1, Z+Y^{V}\right), \\
\Phi_{a}^{i} & =\left(0,\left(F_{a}^{i}\right)^{V}\right), \\
\Gamma_{a, b}^{i} & =\left(0,\left(G_{a, b}^{i}\right)^{V}\right),
\end{aligned}
$$

all seen as vector fields on $\mathbb{R} \times T Q$. We also define $\gamma_{\text {ref }}^{e}(0)=\left(0, \dot{\gamma}_{\text {ref }}(0)\right)$ and, given a smooth function $\lambda: I \rightarrow \mathbb{R}$, we write $\lambda^{V}$ to denote a smooth function on $\mathbb{R} \times T Q$ such that $\lambda^{V}(t, v)=\lambda(t)$ for every $t \in I$ and every $v \in T Q$. In particular, we define

$$
\begin{aligned}
\theta_{a, b}^{i} & =\left(\sqrt{\lambda_{a}^{\epsilon_{1}, \ldots, \epsilon_{i}} \alpha_{a, b}^{l-i-1}}\right)^{V}, \\
\dot{\theta}_{a, b}^{i} & =\left(\frac{\mathrm{d}}{\mathrm{d} t} \sqrt{\lambda_{a}^{\epsilon_{1}, \ldots, \epsilon_{i}} \alpha_{a, b}^{l-i-1}}\right)^{V} .
\end{aligned}
$$

Then, applying (司) and (6),

$$
\begin{aligned}
& \left(t, \dot{\xi}_{0}^{\epsilon_{1}, \ldots, \epsilon_{l}}(t)\right)= \\
& \overrightarrow{\exp } \int_{0}^{t}\left(X^{e}+\sum_{a=1}^{N_{0}} \lambda_{a}^{\epsilon_{1}, \ldots, \epsilon_{l}}(s)\left(0,\left(Z_{a}^{0}\right)^{V}\right)\right) \mathrm{d} s \gamma_{\text {ref }}^{e}(0) \\
& =\overrightarrow{\exp } \int_{0}^{t}\left(X^{e}+\sum_{a=1}^{N_{1}} \lambda_{a}^{\epsilon_{1}, \ldots, \epsilon_{l-1}}(s) \Phi_{a}^{0}\right. \\
& +\frac{1}{\epsilon_{l}} \sum_{a=1}^{N_{1}} \sum_{b=1}^{\hat{N}_{a}^{1}} \psi_{j(a, b)}\left(\frac{s}{\epsilon_{l}}\right) \sqrt{\lambda_{a}^{\epsilon_{1}, \ldots, \epsilon_{l-1}}(s) \alpha_{a, b}^{0}} \\
& \left.\Gamma_{a, b}^{0}\right) \mathrm{d} s \gamma_{\text {ref }}^{e}(0) \\
& =\overrightarrow{\exp } \int_{0}^{t} \frac{1}{\epsilon_{l}} \sum_{a=1}^{N_{1}} \sum_{b=1}^{\hat{N}_{a}^{1}} \psi_{j(a, b)}\left(\frac{s}{\epsilon_{l}}\right) \theta_{a, b}^{l-1} \Gamma_{a, b}^{0} \mathrm{~d} s \circ \\
& \overrightarrow{\exp } \int_{0}^{t}\left(X^{e}+\sum_{a=1}^{N_{1}} \lambda_{a}^{\epsilon_{1}, \ldots, \epsilon_{l-1}}(s) \Phi_{a}^{0}\right. \\
& +\sum_{a=1}^{N_{1}} \sum_{b=1}^{\hat{N}_{a}^{1}} \int_{0}^{s} \frac{1}{\epsilon_{l}} \psi_{j(a, b)}\left(\frac{s_{0}}{\epsilon_{l}}\right) \mathrm{d} s_{0}\left(\theta_{a, b}^{l-1}\left[\Gamma_{a, b}^{0}, X^{e}\right]\right. \\
& \left.-\dot{\theta}_{a, b}^{l-1} \Gamma_{a, b}^{0}\right)-\sum_{a, a^{\prime}=1}^{N_{1}} \sum_{b=1}^{\hat{N}_{a}} \sum_{b_{a^{\prime}}=1}^{\hat{N}^{\prime}} \int_{0}^{s} \frac{1}{\epsilon_{l}} \psi_{j(a, b)}\left(\frac{s_{1}}{\epsilon_{l}}\right) \\
& \int_{0}^{s_{1}} \frac{1}{\epsilon_{l}} \psi_{j\left(a^{\prime}, b^{\prime}\right)}\left(\frac{s_{0}}{\epsilon_{l}}\right) \mathrm{d} s_{0} \mathrm{~d} s_{1} \theta_{a, b}^{l-1} \theta_{a^{\prime}, b^{\prime}}^{l-1} \\
& \left(0,\left\langle G_{a, b}^{0}: G_{a^{\prime}, b^{\prime}}^{0} V^{V}\right)\right) \mathrm{d} s \gamma_{\mathrm{ref}}^{e}(0) .
\end{aligned}
$$

From now on, let us denote by $\mathscr{V}$ any vertical flow, i.e., any flow on $\mathbb{R} \times T Q$ preserving the base point on $Q$.

Notice that, by construction,

$\sum_{a=1}^{N_{1}} \lambda_{a}^{\epsilon_{1}, \ldots, \epsilon_{l-1}}(s) \Phi_{a}^{0}+$

$-\sum_{a, a^{\prime}=1}^{N_{1}} \sum_{b=1}^{\hat{N}_{a}^{1}} \sum_{b^{\prime}=1}^{\hat{N}_{a^{\prime}}^{1}} \Lambda_{T}\left(\psi_{j(a, b)}, \psi_{j\left(a^{\prime}, b^{\prime}\right)}\right)$

$\sqrt{\lambda_{a}^{\epsilon_{1}, \ldots, \epsilon_{l-1}}(s) \lambda_{a}^{\epsilon_{1}, \ldots, \epsilon_{l-1}}(s) \alpha_{a, b}^{0} \alpha_{a^{\prime}, b^{\prime}}^{0}}$

$\left(0,\left\langle G_{a, b}^{0}: G_{a^{\prime}, b^{\prime}}^{0}\right\rangle^{V}\right)=\sum_{a=1}^{N_{1}} \lambda_{a}^{\epsilon_{1}, \ldots, \epsilon_{l}}(s)\left(0,\left(Z_{a}^{1}\right)^{V}\right)$.
Then,

$$
\begin{aligned}
& \left(t, \dot{\xi}_{0}^{\epsilon_{1}, \ldots, \epsilon_{l}}(t)\right)= \\
& \mathscr{V} \circ \overrightarrow{\exp } \int_{0}^{t}\left(X^{e}+\sum_{a=1}^{N_{1}} \lambda_{a}^{\epsilon_{1}, \ldots, \epsilon_{l-1}}(s)\left(0,\left(Z_{a}^{1}\right)^{V}\right)\right. \\
& +\sum_{a=1}^{N_{1}} \sum_{b=1}^{\hat{N}_{a}^{1}} \int_{0}^{s} \frac{1}{\epsilon_{l}} \psi_{j(a, b)}\left(\frac{s_{0}}{\epsilon_{l}}\right) \mathrm{d} s_{0}\left(\theta_{a, b}^{l-1}\left[\Gamma_{a, b}^{0}, X^{e}\right]\right. \\
& \left.-\dot{\theta}_{a, b}^{l-1} \Gamma_{a, b}^{0}\right) \\
& +\sum_{a, a^{\prime}=1}^{N_{1}} \sum_{b=1}^{\hat{N}_{a}^{1}} \sum_{b^{\prime}=1}^{\hat{N}_{a^{\prime}}^{1}}\left(\Lambda_{T}\left(\psi_{j(a, b)}, \psi_{j\left(a^{\prime}, b^{\prime}\right)}\right)\right. \\
& \left.-\int_{0}^{s} \frac{1}{\epsilon_{l}} \psi_{j(a, b)}\left(\frac{s_{1}}{\epsilon_{l}}\right) \int_{0}^{s_{1}} \frac{1}{\epsilon_{l}} \psi_{j\left(a^{\prime}, b^{\prime}\right)}\left(\frac{s_{0}}{\epsilon_{l}}\right) \mathrm{d} s_{0} \mathrm{~d} s_{1}\right) \\
& \left(0,\left\langle G_{a, b}^{0}: G_{a^{\prime}, b^{\prime}}^{0} V^{V}\right) \theta_{a, b}^{l-1} \theta_{a^{\prime}, b^{\prime}}^{l-1}\right) \mathrm{d} s \gamma_{\mathrm{ref}}^{e}(0) .
\end{aligned}
$$

Applying iteratively the same computation as above, one ends up with

$$
\begin{aligned}
& \left(t, \dot{\xi}_{0}^{\epsilon_{1}, \ldots, \epsilon_{l}}(t)\right)= \\
& \mathscr{V} \circ \overrightarrow{\exp } \int_{0}^{t}\left(X^{e}+\lambda_{a}(s)\left(0,\left(Z_{a}^{l}\right)^{V}\right)+\mathscr{T}(s)\right) \mathrm{d} s \gamma_{\mathrm{ref}}^{e}(0),
\end{aligned}
$$

where $\mathscr{T}(s)$ is a sum of terms of the form $\zeta(s) V$ where $V$ is a vector field on $\mathbb{R} \times T Q$ independent of the $\epsilon_{j}$, while $\zeta: I \rightarrow \mathbb{R}$ is smooth, depends on the $\epsilon_{j}$ and is of one of the following four types:

$$
\begin{aligned}
& \zeta_{1}(s)=\int_{0}^{s} \frac{1}{\epsilon_{i}} \psi_{j(a, b)}\left(\frac{s_{0}}{\epsilon_{i}}\right) \mathrm{d} s_{0} \sqrt{\lambda_{a}^{\epsilon_{1}, \ldots, \epsilon_{i-1}}(s) \alpha_{a, b}^{l-i}}, \\
& \zeta_{2}(s)=-\int_{0}^{s} \frac{1}{\epsilon_{i}} \psi_{j(a, b)}\left(\frac{s_{0}}{\epsilon_{i}}\right) \mathrm{d} s_{0} \frac{\mathrm{d}}{\mathrm{d} s} \sqrt{\lambda_{a}^{\epsilon_{1}, \ldots, \epsilon_{i-1}}(s) \alpha_{a, b}^{l-i}}, \\
& \zeta_{3}(s)=\frac{1}{1+\delta_{b c}}\left[2\left(\Lambda_{T}\left(\psi_{j(a, b)}, \psi_{j\left(a^{\prime}, b^{\prime}\right)}\right)\right)\right. \\
& \left.-\left(\int_{0}^{s} \frac{1}{\epsilon_{i}} \psi_{j(a, b)}\left(\frac{s_{0}}{\epsilon_{i}}\right) \mathrm{d} s_{0}\right)\left(\int_{0}^{s} \frac{1}{\epsilon_{i}} \psi_{j\left(a^{\prime}, b^{\prime}\right)}\left(\frac{s_{0}}{\epsilon_{i}}\right) \mathrm{d} s_{0}\right)\right] \\
& \sqrt{\lambda_{a}^{\epsilon_{1}, \ldots, \epsilon_{i-1}}(s) \lambda_{a^{\prime}}^{\epsilon_{1}, \ldots, \epsilon_{i-1}}(s) \alpha_{a, b}^{l-i} \alpha_{a^{\prime}, b^{\prime}}^{l-i}}
\end{aligned}
$$

for $i=1, \ldots, l$, or

$$
\begin{aligned}
& \zeta_{4}(s)=-\left(\int_{0}^{s} \frac{1}{\epsilon_{j}} \psi_{j(a, b)}\left(\frac{s_{0}}{\epsilon_{j}}\right) \mathrm{d} s_{0}\right) \\
& \left(\int_{0}^{s} \frac{1}{\epsilon_{i}} \psi_{j\left(a^{\prime}, b^{\prime}\right)}\left(\frac{s_{0}}{\epsilon_{i}}\right) \mathrm{d} s_{0}\right) \\
& \sqrt{\lambda_{a}^{\epsilon_{1}, \ldots, \epsilon_{j-1}}(s) \lambda_{a^{\prime}}^{\epsilon_{1}, \ldots, \epsilon_{i-1}}(s) \alpha_{a, b}^{l-j} \alpha_{a^{\prime}, b^{\prime}}^{l-i}}
\end{aligned}
$$

for $i>j, i, j \in\{1, \ldots, l\}$.

According to Lemma 2.4, the theorem is proved if we show that, for every $\zeta$ of one of the four types introduced above, $\int_{0}^{t} \zeta(s) \mathrm{d} s$ converges to zero uniformly with respect to $t \in I$ as $\epsilon$ goes to zero.

This can be done by applying Lemma 5.1. Taking for instance

$$
f(\tau, s)=\int_{0}^{\tau} \psi_{j(a, b)}\left(s_{0}\right) \mathrm{d} s_{0} \sqrt{\lambda_{a}^{\epsilon_{1}, \ldots, \epsilon_{i-1}}(s) \alpha_{a, b}^{l-i}},
$$

and $\hat{\epsilon}=\epsilon_{i}$ leads to

$$
\int_{0}^{t} \zeta_{1}(s) \mathrm{d} s \leq C \frac{\epsilon_{i}}{\sqrt[2 \sqrt[i-1]{\epsilon_{1}} \sqrt[2^{i-2}]{\epsilon_{2}} \cdots \sqrt[4]{\epsilon_{i-2}}]{\epsilon_{i-1}^{\frac{3}{2}}}} .
$$

Similarly,

$$
\int_{0}^{t} \zeta_{2}(s) \mathrm{d} s \leq C \frac{\epsilon_{i}}{\sqrt[2 \sqrt[i-1]{\epsilon_{1}} \sqrt[2^{i-2}]{\epsilon_{2}} \cdots \sqrt[4]{\epsilon_{i-2}}]{\epsilon_{i-1}^{\frac{5}{2}}}} .
$$


Taking

$$
\begin{aligned}
f(\tau, s)= & \left(\int_{0}^{\tau} \psi_{j(a, b)}\left(s_{0}\right) \mathrm{d} s_{0}\right)\left(\int_{0}^{\tau} \psi_{j\left(a^{\prime}, b^{\prime}\right)}\left(s_{0}\right) \mathrm{d} s_{0}\right) \\
& \sqrt{\lambda_{a}^{\epsilon_{1}, \ldots, \epsilon_{i-1}}(s) \lambda_{a^{\prime}}^{\epsilon_{1}, \ldots, \epsilon_{i-1}}(s) \alpha_{a, b}^{l-i} \alpha_{a^{\prime}, b^{\prime}}^{l-i}}
\end{aligned}
$$

we obtain

$$
\int_{0}^{t} \zeta_{3}(s) \mathrm{d} s \leq C \frac{\epsilon_{i}}{\sqrt[2^{i-2}]{\epsilon_{1}} \sqrt[2^{i-3}]{\epsilon_{2}} \cdots \sqrt{\epsilon_{i-2}} \epsilon_{i-1}^{2}} .
$$

Finally, with

$$
\begin{aligned}
& f(\tau, s)=\left(\int_{0}^{s / \epsilon_{j}} \psi_{j(a, b)}\left(s_{0}\right) \mathrm{d} s_{0}\right)\left(\int_{0}^{\tau} \psi_{j\left(a^{\prime}, b^{\prime}\right)}\left(s_{0}\right) \mathrm{d} s_{0}\right) \\
& \sqrt{\lambda_{a}^{\epsilon_{1}, \ldots, \epsilon_{j-1}}(s) \lambda_{a^{\prime}}^{\epsilon_{1}, \ldots, \epsilon_{i-1}}(s) \alpha_{a, b}^{l-j} \alpha_{a^{\prime}, b^{\prime}}^{l-i}}
\end{aligned}
$$

we get

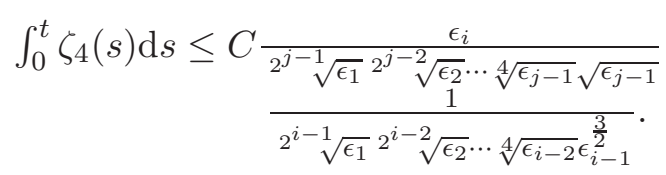

Notice that the upper bound for $\int_{0}^{t} \zeta_{2}(s) \mathrm{d} s$ is the one growing faster as $\epsilon$ goes to zero. Indeed, in order to compare it with the one for $\int_{0}^{t} \zeta_{3}(s) \mathrm{d} s$ it suffices to notice that

$$
\begin{aligned}
& \frac{2^{i-1} \sqrt{\epsilon_{1}} 2^{i-2} \sqrt{\epsilon_{2}} \cdots \sqrt[4]{\epsilon_{i-2}} \epsilon_{i-1}^{\frac{5}{2}}}{2^{i-2}{ }_{\epsilon_{1}} \sqrt[2]{\epsilon_{2} \cdots \sqrt{\epsilon_{i-2}} \epsilon_{i-1}^{2}}}= \\
& \sqrt{\frac{\epsilon_{i-1}}{\epsilon_{i-2}}} \sqrt[4]{\frac{\epsilon_{i-2}}{\epsilon_{i-3}}} \cdots \sqrt[2 \sqrt[i-2]{\frac{\epsilon_{2}}{\epsilon_{1}}}]{2^{i-1}} \sqrt{\epsilon_{1}}
\end{aligned}
$$

converges to zero as $\epsilon$ goes to zero.

Hence, for each $\zeta$ as above, there exists $i=1, \ldots, l$ such that

$$
\int_{0}^{t} \zeta(s) \mathrm{d} s \leq C \frac{\epsilon_{i}}{\sqrt[2-1]{\epsilon_{1}} \sqrt[2^{i-2}]{\epsilon_{2}} \cdots \sqrt[4]{\epsilon_{i-2}} \epsilon_{i-1}^{\frac{5}{2}}}
$$

and we are left to notice that

$$
\begin{aligned}
& \frac{\epsilon_{i}}{2 \sqrt[i-1]{\epsilon_{1}} 2^{i-2} \sqrt{\epsilon_{2}} \cdots \sqrt[4]{\epsilon_{i-2}} \epsilon_{i-1}^{\frac{5}{2}}}= \\
& \frac{\epsilon_{i}}{\epsilon_{i-1}^{3}} \sqrt{\frac{\epsilon_{i-1}}{\epsilon_{i-2}}} \sqrt[4]{\frac{\epsilon_{i-2}}{\epsilon_{i-3}}} \cdots \sqrt[2]{\sqrt[i-2]{\frac{\epsilon_{2}}{\epsilon_{1}}}} 2^{i-1} \sqrt{\epsilon_{1}}
\end{aligned}
$$

tends to zero as $\epsilon$ goes to zero.

\section{Remark}

5.3. The

hypothesis

$\lim \sup _{\epsilon \rightarrow 0} \eta_{i}(\epsilon) / \epsilon^{3}<\infty$ has been chosen, in the statement of Theorem 5.2, because of its simplicity. However, we can weaken it by requiring that $\lim \sup _{\epsilon \rightarrow 0} \eta_{i}(\epsilon) / \epsilon^{\frac{5}{2}+a}<\infty$ with $a=\frac{\sqrt{5}}{2}-1 \simeq 0.12$. Indeed, with this choice of $a$,

$$
\begin{aligned}
& \frac{\epsilon_{j}^{a}}{\sqrt[2^{j}]{\epsilon_{1}} \sqrt[2^{j-1}]{\epsilon_{2} \cdots \sqrt[4]{\epsilon_{j-1}}}}=\left(\frac{\epsilon_{j}}{\epsilon_{j-1}^{\frac{5}{2}+a}}\right)^{a} \\
& \left(\frac{\epsilon_{j-1}^{a}}{\sqrt[2^{j-1}]{\epsilon_{1}} \sqrt[2^{j-2}]{\epsilon_{2} \cdots \sqrt[4]{\epsilon_{j-2}}}}\right)^{\frac{1}{2}},
\end{aligned}
$$

so that

$$
\begin{aligned}
& \frac{\epsilon_{i}}{\sqrt[2^{i-1}]{\epsilon_{1}} 2^{i-2} \sqrt{\epsilon_{2}} \cdots \sqrt[4]{\epsilon_{i-2}} \epsilon_{i-1}^{\frac{5}{2}}}= \\
& \frac{\epsilon_{i}}{\epsilon_{i-1}^{\frac{5}{2}+a}}\left(\frac{\epsilon_{i-1}}{\epsilon_{i-2}^{\frac{5}{2}+a}}\right)^{a}\left(\frac{\epsilon_{i-2}}{\frac{5}{\epsilon_{i-3}^{\frac{5}{2}+a}}}\right)^{\frac{a}{2}} \cdots\left(\frac{\epsilon_{2}}{\epsilon_{1}^{\frac{5}{2}+a}}\right)^{\frac{a}{2^{i-3}}} \epsilon_{1}^{\frac{a}{2^{2-2}}} .
\end{aligned}
$$

Hence, each $\int_{0}^{t} \zeta(s) \mathrm{d} s$ goes to zero uniformly with respect to $t \in I$ as $\epsilon$ tends to zero.

\subsection{Case $\mathscr{Z}$}

Analogously to Section 5.1, the aim is to provide an algorithmic implementation of the results obtained in Section 14 about the existence of controls yielding tracking but this time under the hypotheses of Corollary 4.7. Instead of adopting the algorithmic scheme on which the proof of Theorem 4.4 is based, as done in Section 5.1, we rely here on the iteration of the scheme proposed in [5] and recalled in Theorem 3.3 (see also Remark 4.3). The advantage is that, under the more restrictive hypotheses of Corollary 4.7, we can base the iteration scheme on the $\Lambda_{T}$-orthonormal and zero-mean sequence defined in (8) using trigonometric functions, which is more convenient for numerical implementation than the sequence $\left(\psi_{j}\right)_{j \in \mathbb{N}}$ constructed in the previous section.

We start from a parameterization

$$
\nabla_{\dot{\gamma}_{\mathrm{ref}}(t)} \dot{\gamma}_{\mathrm{ref}}(t)-Y\left(t, \dot{\gamma}_{\mathrm{ref}}(t)\right)=\sum_{a=1}^{N_{l}} \lambda_{a}(t) Z_{a}^{l}\left(\gamma_{\mathrm{ref}}(t)\right)
$$

of $\gamma_{\text {ref }}: I \rightarrow Q$, with $Z_{a}^{l} \in \mathscr{Z}_{l}$ and $\lambda_{a}$ smooth on $I$ for every $a=1, \ldots, N_{l}$. (For the definition of $\mathscr{Z}_{l}$, see (17).) As in Section 5.1, we can construct algorithmically a $l$-parameter family of admissible trajectories $\xi^{\epsilon_{1}, \ldots, \epsilon_{l}}$ of $\Sigma$ with $\epsilon_{1}, \ldots, \epsilon_{l}>0$ such that $\xi_{l}=\gamma_{\text {ref }}$, the uniform limit in (20) exists for every $i=1, \ldots, l$ and every $\epsilon_{1}, \ldots, \epsilon_{l-i}>0$, and, instead of (21), it satisfies

$$
\begin{aligned}
& \nabla_{\dot{\xi}_{i}^{\epsilon_{1}, \ldots, \epsilon_{l-i}}(t)} \dot{\xi}_{i}^{\epsilon_{1}, \ldots, \epsilon_{l-i}}(t)-Y\left(t, \dot{\xi}_{i}^{\epsilon_{1}, \ldots, \epsilon_{l-i}}(t)\right) \\
& \quad \in \operatorname{span}_{\mathbb{R}}\left(\mathscr{Z}_{i}\left(\xi_{i}^{\epsilon_{1}, \ldots, \epsilon_{l-i}}(t)\right)\right) .
\end{aligned}
$$

The algorithm works by applying, at each step, the construction of Theorem 3.3 with $\mathscr{Z}_{i}$ as $\mathscr{Y}$ (see Remark 4.3). Then by backward recursion on $i$, the 
solutions to

$$
\begin{aligned}
& \nabla_{\dot{\xi}_{i}^{\epsilon_{1}}, \ldots, \epsilon_{l-i}(t)} \dot{\xi}_{i}^{\epsilon_{1}, \ldots, \epsilon_{l-i}}(t)-Y\left(t, \dot{\xi}_{i}^{\epsilon_{1}, \ldots, \epsilon_{l-i}}(t)\right) \\
& =\sum_{a=1}^{N_{i}} \lambda_{a}^{\epsilon_{1}, \ldots, \epsilon_{l-i}}(t) Z_{a}^{i-1}\left(\xi_{i}^{\epsilon_{1}, \ldots, \epsilon_{l-i}}(t)\right) \\
& +\sum_{b, c=1, b<c}^{N_{i}} \lambda_{b, c}^{\epsilon_{1}, \ldots, \epsilon_{l-i}}(t)\left\langle Z_{b}^{i-1}: Z_{c}^{i-1}\right\rangle\left(\xi_{i}^{\epsilon_{1}, \ldots, \epsilon_{l-i}}(t)\right)
\end{aligned}
$$

for $Z_{a}^{i-1}, Z_{b}^{i-1}, Z_{c}^{i-1} \in \mathscr{Z}_{i-1}$ are trackable by solutions to

$$
\begin{aligned}
& \nabla_{\dot{\xi}_{i-1}^{\epsilon_{1}, \ldots, \epsilon_{l-i+1}}(t)} \dot{\xi}_{i-1}^{\epsilon_{1}, \ldots, \epsilon_{l-i+1}}(t)-Y\left(t, \dot{\xi}_{i}^{\epsilon_{1}, \ldots, \epsilon_{l-i+1}}(t)\right) \\
& =\sum_{a=1}^{N_{i-1}}\left(u_{\mathrm{slow}, a}^{\epsilon_{1}, \ldots, \epsilon_{l-i}}(t)\right. \\
& \left.+\frac{1}{\epsilon_{l-i+1}} u_{\mathrm{OSc}, a}^{\epsilon_{1}, \ldots, \epsilon_{l-i}}\left(t / \epsilon_{l-i+1}, t\right)\right) Z_{a}^{i-1}\left(\xi_{i-1}^{\epsilon_{1}, \ldots, \epsilon_{l-i+1}}(t)\right)
\end{aligned}
$$

where $u_{\text {slow. } a}^{\epsilon_{1}, \ldots, \epsilon_{l-i}}$ and $u_{\text {osc }, a}^{\epsilon_{1}, \ldots, \epsilon_{l-i}}$ are constructed as in Theorem 3.3. Notice that

$$
u_{\text {slow }, a}^{\epsilon_{1}, \ldots, \epsilon_{l-i}}(t)+\frac{1}{\epsilon_{l-i+1}} u_{\mathrm{osc}, a}^{\epsilon_{1}, \ldots, \epsilon_{l-i}}\left(t / \epsilon_{l-i+1}, t\right)
$$

plays the role of a $\lambda_{\hat{a}}^{\epsilon_{1}, \ldots, \epsilon_{l-i+1}}(t)$ or a $\lambda_{\hat{b}, \hat{c}}^{\epsilon_{1}, \ldots, \epsilon_{l-i+1}}(t)$ at the next step.

We have

$$
\begin{gathered}
\left\|u_{\text {slow }, a}^{\epsilon_{1}, \ldots, \epsilon_{l-i}}\right\|_{\infty} \leq C \max _{b, c}\left(\left\|\lambda_{b}^{\epsilon_{1}, \ldots, \epsilon_{l-i}}\right\|_{\infty},\left\|\lambda_{b, c}^{\epsilon_{1}, \ldots, \epsilon_{l-i}}\right\|_{\infty}^{2}\right), \\
\left\|u_{\mathrm{osc}, a}^{\epsilon_{1}, \ldots, \epsilon_{l-i}}\right\|_{\infty} \leq C \max _{b, c}\left(\left\|\lambda_{b, c}^{\epsilon_{1}, \ldots, \epsilon_{l-i}}\right\|_{\infty}\right) .
\end{gathered}
$$

Given $\alpha, \beta:[0, T] \times I \rightarrow \mathbb{R}$, define $\Lambda_{T}$ as

$$
\begin{aligned}
& \Lambda_{T}(t, \alpha, \beta)= \\
& \quad \frac{1}{2 T} \int_{0}^{T}\left(\int_{0}^{\tau} \alpha(s, t) \mathrm{d} s\right)\left(\int_{0}^{\tau} \beta(s, t) \mathrm{d} s\right) \mathrm{d} \tau .
\end{aligned}
$$

The construction of $u_{\text {slow }}$ and $u_{\text {osc }}$ is such that

$$
\begin{aligned}
& \sum_{a=1}^{N_{i-1}} u_{\text {slow }, a}^{\epsilon_{1}, \ldots, \epsilon_{l-i}}(t) Z_{a}^{i-1} \\
& -\sum_{b, c=1}^{N_{i-1}} \Lambda_{T}\left(t, u_{\mathrm{osc}, b}^{\epsilon_{1}, \ldots, \epsilon_{l-i}}, u_{\mathrm{osc}, c}^{\epsilon_{1}, \ldots, \epsilon_{l-i}}\right)\left\langle Z_{b}^{i-1}: Z_{c}^{i-1}\right\rangle \\
& =\sum_{a=1}^{N_{i-1}} \lambda_{a}^{\epsilon_{1}, \ldots, \epsilon_{l-i}}(t) Z_{a}^{i-1} \\
& +\sum_{b, c=1, b<c}^{N_{i-1}} \lambda_{b, c}^{\epsilon_{1}, \ldots, \epsilon_{l-i}}(t)\left\langle Z_{b}^{i-1}: Z_{c}^{i-1}\right\rangle
\end{aligned}
$$

(see [5). As in Section 5.1, our aim is here to quantify the relations in (24). We will use the same notations for $\eta_{i}$ and $\hat{\eta}_{i}$ as in Section 5.1. The notion of being a one-parameter tracking control law for $\gamma_{\text {ref }}$ is again defined through (25).
Theorem 5.4. Let $\Sigma=\left(Q, \nabla, Y, \mathscr{Y}, \mathbb{R}^{k}\right)$ be a FACCS. Let $\mathscr{Z}_{i}, i \in \mathbb{N}_{0}$, be defined as in (17) satisfying the same hypotheses as in Corollary 4.7. Fix a reference trajectory $\gamma_{\mathrm{ref}} \in \mathcal{C}^{\infty}(I, Q)$ and assume that there exists $l \in \mathbb{N}$ such that

$$
\nabla_{\dot{\gamma}_{\text {ref }}(t)} \dot{\gamma}_{\text {ref }}(t)-Y\left(t, \dot{\gamma}_{\text {ref }}(t)\right) \in \mathscr{Z}_{l}\left(\gamma_{\text {ref }}(t)\right)
$$

for every $t \in I$. Construct $\xi^{\epsilon_{1}, \ldots, \epsilon_{l}}, u^{\epsilon_{1}, \ldots, \epsilon_{l}}$ and $\hat{\eta}_{i}$ as above. If $\eta_{i}:(0,+\infty) \rightarrow(0,+\infty)$ satisfies $\lim \sup _{\epsilon \rightarrow 0} \eta_{i}(\epsilon) / \epsilon^{4}<\infty$ for every $i=2, \ldots, l$, then $\epsilon \mapsto u^{\hat{\eta}_{1}(\epsilon), \ldots, \hat{\eta}_{l}(\epsilon)}$ is a one-parameter tracking control law for $\gamma_{\mathrm{ref}}$.

Proof. The first step of the proof consists in estimating the order with respect to $\epsilon$ of the $\mathrm{L}^{\infty}{ }_{\text {-norm }}$ of the time-dependent parameters appearing in the parameterization (27). Once more we write $\epsilon_{i}$ for $\hat{\eta}_{i}(\epsilon)$. By induction it can be proved that

$$
\left\|\partial_{2}^{m} u_{\mathrm{osc}, a}^{\epsilon_{1}, \ldots, \epsilon_{i}}\right\|_{\infty} \leq C\left(\epsilon_{1} \cdots \epsilon_{i-1} \epsilon_{i}^{m+1}\right)^{-1}
$$

for every $m \in \mathbb{N}_{0}$.

Consider, as in the proof of Theorem 5.2, the extended system on $\mathbb{R} \times T Q$ associated with (3). In the following computations we write

$$
\begin{aligned}
V_{a}^{i} & =\left(0,\left(Z_{a}^{i}\right)^{V}\right), \\
W_{b, c}^{i} & =\left(0,\left\langle Z_{b}^{i}: Z_{c}^{i}\right\rangle^{V}\right) .
\end{aligned}
$$

We also write $u_{\text {osc }, a}^{\epsilon_{1}, \ldots, \epsilon_{i}}(\sigma)$ to denote $\left(u_{\text {osc }, a}^{\epsilon_{1}, \ldots, \epsilon_{i}}(\sigma, \cdot)\right)^{V}$, i.e., $u_{\mathrm{osc}, a}^{\epsilon_{1}, \ldots, \epsilon_{i}}(\sigma)$ is the smooth function on $\mathbb{R} \times T Q$ such that

$$
u_{\mathrm{osc}, a}^{\epsilon_{1}, \ldots, \epsilon_{i}}(\sigma)(t, v)=u_{\mathrm{osc}, a}^{\epsilon_{1}, \ldots, \epsilon_{i}}(\sigma, t) .
$$

Then, applying (5) and (6),

$$
\begin{aligned}
& \left(t, \dot{\xi}_{0}^{\epsilon_{1}, \ldots, \epsilon_{l}}(t)\right)=\overrightarrow{\exp } \int_{0}^{t}\left(X^{e}\right. \\
& +\sum_{a=1}^{N_{0}} u_{\text {slow }, a}^{\epsilon_{1}, \ldots, \epsilon_{l-1}}(s) V_{a}^{0} \\
& \left.+\frac{1}{\epsilon_{l}} \sum_{a=1}^{N_{0}} u_{\mathrm{osc}, a}^{\epsilon_{1}, \ldots, \epsilon_{l-1}}\left(s / \epsilon_{l}, s\right) V_{a}^{0}\right) \mathrm{d} s \gamma_{\mathrm{ref}}^{e}(0) \\
& =\overrightarrow{\exp } \int_{0}^{t} \frac{1}{\epsilon_{l}} \sum_{a=1}^{N_{0}} u_{\mathrm{osc}, a}^{\epsilon_{1}, \ldots, \epsilon_{l-1}}\left(s / \epsilon_{l}\right) V_{a}^{0} \mathrm{~d} s \circ \\
& \overrightarrow{\exp } \int_{0}^{t}\left(X^{e}+\sum_{a=1}^{N_{0}} u_{\text {slow }, a}^{\epsilon_{1}, \ldots, \epsilon_{l-1}}(s) V_{a}^{0}\right. \\
& +\sum_{a=1}^{N_{0}} \int_{0}^{s} \frac{1}{\epsilon_{l}} u_{\mathrm{osc}, a}^{\epsilon_{1}, \ldots, \epsilon_{l-1}}\left(s_{0} / \epsilon_{l}\right) \mathrm{d} s_{0}\left[V_{a}^{0}, X^{e}\right] \\
& -\sum_{a=1}^{N_{0}} \int_{0}^{s} \frac{1}{\epsilon_{l}} \partial_{2} u_{\mathrm{osc}, a}^{\epsilon_{1}, \ldots, \epsilon_{l-1}}\left(s_{0} / \epsilon_{l}\right) \mathrm{d} s_{0} V_{a}^{0} \\
& -\sum_{b, c=1}^{N_{0}} \int_{0}^{s} \frac{1}{\epsilon_{l}} u_{\mathrm{osc}, b}^{\epsilon_{1}, \ldots, \epsilon_{l-1}}\left(s_{1} / \epsilon_{l}\right) \\
& \left.\int_{0}^{s_{1}} \frac{1}{\epsilon_{l}} u_{\text {osc }, c}^{\epsilon_{1}, \ldots, \epsilon_{l-1}}\left(s_{0} / \epsilon_{l}\right) \mathrm{d} s_{0} \mathrm{~d} s_{1} W_{b, c}^{0}\right) \mathrm{d} s \gamma_{\text {ref }}^{e}(0)
\end{aligned}
$$


Denoting by $\mathscr{V}$ any vertical flow, we have

$$
\begin{aligned}
& \left(t, \dot{\xi}_{0}^{\epsilon_{1}, \ldots, \epsilon_{l}}(t)\right)=\mathscr{V} \circ \overrightarrow{\exp } \int_{0}^{t}\left(X^{e}\right. \\
& +\sum_{a=1}^{N_{0}} \lambda_{a}^{\epsilon_{1}, \ldots, \epsilon_{l-1}}(s) V_{a}^{0} \\
& +\sum_{b, c=1, b<c}^{N_{0}} \lambda_{b, c}^{\epsilon_{1}, \ldots, \epsilon_{l-1}}(s) W_{b, c}^{0} \\
& +\int_{0}^{s} \frac{1}{\epsilon_{l}} \sum_{a=1}^{N_{0}} u_{\mathrm{osc}, a}^{\epsilon_{1}, \ldots, \epsilon_{l-1}}\left(s_{0} / \epsilon_{l}, s\right) \mathrm{d} s_{0}\left[V_{a}^{0}, X^{e}\right] \\
& -\sum_{a=1}^{N_{0}} \int_{0}^{s} \frac{1}{\epsilon_{l}} \partial_{2} u_{\mathrm{osc}, a}^{\epsilon_{1}, \ldots, \epsilon_{l-1}}\left(s_{0} / \epsilon_{l}, s\right) \mathrm{d} s_{0} V_{a}^{0} \\
& +\sum_{b, c=1}^{N_{0}}\left(\Lambda_{T}\left(s, u_{\mathrm{osc}, b}^{\epsilon_{1}, \ldots, \epsilon_{l-1}}, u_{\mathrm{osc}, c}^{\epsilon_{1}, \ldots, \epsilon_{l-1}}\right)-\right. \\
& \int_{0}^{s} \frac{1}{\epsilon_{l}} u_{\mathrm{osc}, b}^{\epsilon_{1}, \ldots, \epsilon_{l-1}}\left(s_{1} / \epsilon_{l}, s\right) \int_{0}^{s_{1}} \frac{1}{\epsilon_{l}} u_{\mathrm{osc}, c}^{\epsilon_{1}, \ldots, \epsilon_{l-1}}\left(s_{0} / \epsilon_{l}, s\right) \\
& \left.\left.\mathrm{d} s_{0} \mathrm{~d} s_{1}\right) W_{b, c}^{0}\right) \mathrm{d} s \gamma_{\text {ref }}^{e}(0) \text {. }
\end{aligned}
$$

Noticing that

$$
\begin{aligned}
& \sum_{a=1}^{N_{0}} \lambda_{a}^{\epsilon_{1}, \ldots, \epsilon_{l-1}}(s) V_{a}^{0}+\sum_{b, c=1}^{N_{0}}\left(\lambda_{b, c}^{\epsilon_{1}, \ldots, \epsilon_{l-1}}(s) W_{b, c}^{0}\right. \\
& =\sum_{\hat{a}=1}^{N_{1}}\left(u_{\text {slow }, \hat{a}}^{\epsilon_{1}, \ldots, \epsilon_{l-2}}(s)+\frac{1}{\epsilon_{l-1}} u_{\text {osc }, \hat{a}}^{\epsilon_{1}, \ldots,,_{l-2}}\left(s / \epsilon_{l-1}, s\right)\right) V_{\hat{a}}^{1}
\end{aligned}
$$

and applying iteratively the same computation as above, one ends up with

$$
\begin{aligned}
& \left(t, \dot{\xi}_{0}^{\epsilon_{1}, \ldots, \epsilon_{l}}(t)\right)= \\
& \mathscr{V} \circ \overrightarrow{\exp } \int_{0}^{t}\left(X^{e}+\lambda_{a}(s) V_{a}^{l}+\mathscr{T}(s)\right) \mathrm{d} s \gamma_{\mathrm{ref}}^{e}(0),
\end{aligned}
$$

where $\mathscr{T}(s)$ is a sum of terms of the form $\zeta(s) V$ where $V \in \mathscr{X}(\mathbb{R} \times T Q)$ is independent of the $\epsilon_{j}$, while $\zeta \in \mathcal{C}^{\infty}(I, \mathbb{R})$ is of one of the following four types:

$$
\begin{aligned}
& \zeta_{1}=\int_{0}^{s} \frac{1}{\epsilon_{i}} u_{\text {osc, }}^{\epsilon_{1}, \ldots, \epsilon_{i-1}}\left(s_{0} / \epsilon_{i}, s\right) \mathrm{d} s_{0}, \\
& \zeta_{2}=-\int_{0}^{s} \frac{1}{\epsilon_{i}} \partial_{2} u_{\text {osc }, a}^{\epsilon_{1}, \ldots, \epsilon_{i-1}}\left(s_{0} / \epsilon_{i}, s\right) \mathrm{d} s_{0}, \\
& \zeta_{3}=\frac{1}{1+\delta_{b c}}\left[2 \Lambda_{T}\left(s, u_{\mathrm{osc}, b}^{\epsilon_{1}, \ldots, \epsilon_{i-1}}, u_{\mathrm{OSc}, c}^{\epsilon_{1}, \ldots, \epsilon_{i-1}}\right)\right. \\
& -\left(\int_{0}^{s} \frac{1}{\epsilon_{i}} u_{\mathrm{Osc}, b}^{\epsilon_{1}, \ldots, \epsilon_{i-1}}\left(s_{0} / \epsilon_{i}, s\right) \mathrm{d} s_{0}\right) \\
& \left.\left(\int_{0}^{s} \frac{1}{\epsilon_{i}} u_{\mathrm{Osc}, c}^{\epsilon_{1}, \ldots, \epsilon_{i-1}}\left(s_{0} / \epsilon_{i}, s\right) \mathrm{d} s_{0}\right)\right],
\end{aligned}
$$

for $i=1, \ldots, l$, and

$$
\begin{aligned}
& \zeta_{4}=-\left(\int_{0}^{s} \frac{1}{\epsilon_{j}} u_{\mathrm{osc}, b}^{\epsilon_{1}, \ldots, \epsilon_{j-1}}\left(s_{0} / \epsilon_{j}, s\right) \mathrm{d} s_{0}\right) \\
& \left(\int_{0}^{s} \frac{1}{\epsilon_{i}} u_{\mathrm{osc}, c}^{\epsilon_{1}, \ldots, \epsilon_{i-1}}\left(s_{0} / \epsilon_{i}, s\right) \mathrm{d} s_{0}\right),
\end{aligned}
$$

for $i>j, i, j \in\{1, \ldots, l\}$.

We are left to prove that every $\int_{0}^{t} \zeta(s) \mathrm{d} s$ converges to zero uniformly with respect to $t$ as $\epsilon$ goes to zero (Lemma 2.4).

Applying Lemma 5.1 with

$$
f(\tau, s)=\int_{0}^{\tau} u_{\mathrm{osc}, a}^{\epsilon_{1}, \ldots, \epsilon_{i-1}}\left(s_{0}, s\right) \mathrm{d} s_{0}
$$

and $\hat{\epsilon}=\epsilon_{i}$ leads to

$$
\int_{0}^{t} \zeta_{1}(s) \mathrm{d} s \leq C \epsilon_{i}\left(\epsilon_{1} \cdots \epsilon_{i-2} \epsilon_{i-1}^{2}\right)^{-1} .
$$

Similarly,

$$
\int_{0}^{t} \zeta_{2}(s) \mathrm{d} s \leq C\left(\epsilon_{1} \cdots \epsilon_{i-2} \epsilon_{i-1}^{3}\right)^{-1} \epsilon_{i} .
$$

Taking

$$
\begin{aligned}
& f(\tau, s)=\left(\int_{0}^{\tau} u_{\mathrm{osc}, b}^{\epsilon_{1}, \ldots, \epsilon_{i-1}}\left(s_{0}, s\right) \mathrm{d} s_{0}\right) \\
& \left(\int_{0}^{\tau} u_{\mathrm{osc}, c}^{\epsilon_{1}, \ldots, \epsilon_{i-1}}\left(s_{0}, s\right) \mathrm{d} s_{0}\right),
\end{aligned}
$$

we obtain

$$
\int_{0}^{t} \zeta_{3}(s) \mathrm{d} s \leq C\left(\epsilon_{1} \cdots \epsilon_{i-2}\right)^{-2} \epsilon_{i-1}^{-3} \epsilon_{i} .
$$

Finally, with

$$
\begin{aligned}
& f(\tau, s)=\left(\int_{0}^{s / \epsilon_{j}} u_{\mathrm{osc}, b}^{\epsilon_{1}, \ldots, \epsilon_{j-1}}\left(s_{0}, s\right) \mathrm{d} s_{0}\right) \\
& \quad\left(\int_{0}^{\tau} u_{\mathrm{osc}, c}^{\epsilon_{1}, \ldots, \epsilon_{i-1}}\left(s_{0}, s\right) \mathrm{d} s_{0}\right),
\end{aligned}
$$

we have

$\int_{0}^{t} \zeta_{4}(s) \mathrm{d} s \leq C\left(\epsilon_{1} \cdots \epsilon_{j-2} \epsilon_{j-1}\right)^{-1}\left(\epsilon_{1} \cdots \epsilon_{i-2} \epsilon_{i-1}^{2}\right)^{-1} \epsilon_{i}$.

Hence, each $\zeta$ satisfies

$$
\int_{0}^{t} \zeta(s) \mathrm{d} s \leq \frac{C \epsilon_{i}}{\left(\epsilon_{1} \cdots \epsilon_{i-2}\right)^{2} \epsilon_{i-1}^{3}}=\frac{C \epsilon_{i}}{\epsilon_{i-1}^{4}} \frac{\epsilon_{i-1}}{\left(\epsilon_{1} \cdots \epsilon_{i-2}\right)^{2}}
$$

and it is easy to prove by recurrence that $\epsilon_{i-1}\left(\epsilon_{1} \cdots \epsilon_{i-2}\right)^{-2}$ tends to zero as $\epsilon$ goes to zero.

Remark 5.5. As in Remark 5.3, the hypothesis $\lim \sup _{\epsilon \rightarrow 0} \eta_{i}(\epsilon) / \epsilon^{4}<\infty$ in the statement of Theorem 5.4 can be weakened by requiring that $\limsup \sup _{\epsilon \rightarrow 0} \eta_{i}(\epsilon) / \epsilon^{3+a}<\infty$ with $a=\sqrt{3}-1 \simeq 0.73$. Indeed, with this choice of $a$,

$$
\frac{\epsilon_{i}^{a}}{\epsilon_{1}^{2} \epsilon_{2}^{2} \cdots \epsilon_{i-1}^{2}}=\left(\frac{\epsilon_{i}}{\epsilon_{i-1}^{3+a}}\right)^{a}\left(\frac{\epsilon_{i-1}^{a}}{\epsilon_{1}^{2} \cdots \epsilon_{i-2}^{2}}\right),
$$

so that

$$
\begin{aligned}
& \frac{\epsilon_{i}}{\epsilon_{1}^{2} \cdots \epsilon_{i-2}^{2} \epsilon_{i-1}^{3}}= \\
& \frac{\epsilon_{i}}{\epsilon_{i-1}^{3+a}}\left(\frac{\epsilon_{i-1}}{\epsilon_{i-2}^{3+a}}\right)^{a}\left(\frac{\epsilon_{i-2}}{\epsilon_{i-3}^{3+a}}\right)^{a} \cdots\left(\frac{\epsilon_{2}}{\epsilon_{1}^{3+a}}\right)^{a} \epsilon_{1}^{a} .
\end{aligned}
$$

Hence, each $\int_{0}^{t} \zeta(s) \mathrm{d} s$ goes to zero uniformly with respect to $t \in I$ as $\epsilon$ tends to zero.

Under special assumptions the relations in (24) that have been quantified in Theorem 5.4 can be reduced up to $\lim _{\epsilon \rightarrow 0} \eta_{i}(\epsilon) / \epsilon^{2}=0$ as stated in the following corollary and illustrated in the numerical simulation included in Section 5.2.1. The required assumptions are stated in terms of the number of steps in the algorithm and of the coefficients providing the parametrization of the reference trajectory.

Corollary 5.6. Let $\Sigma=\left(Q, \nabla, Y, \mathscr{Y}, \mathbb{R}^{k}\right)$ be a FACCS. Let $\mathscr{Z}_{i}, i \in \mathbb{N}_{0}$, be defined as in (17) and assume that $\operatorname{span}_{\mathbb{R}} \mathscr{Z}_{2}(q)=T_{q} Q$ for all $q \in Q$ 
and that for each $i \in\{0,1\}$ and each $Z \in \mathscr{Z}_{i}$, $\langle Z: Z\rangle(q) \in \operatorname{span}_{\mathbb{R}}\left(\mathscr{Z}_{i}(q)\right)$. Fix a reference trajectory $\gamma_{\mathrm{ref}} \in \mathcal{C}^{\infty}(I, Q)$ such that the coefficients $\lambda_{a}$ associated with $\gamma_{\mathrm{ref}}$ as in (20) are constant functions. Construct $\xi^{\epsilon_{1}, \epsilon_{2}}, u^{\epsilon_{1}, \epsilon_{2}}$ and $\hat{\eta}_{i}$ as above. If $\eta_{2}:(0,+\infty) \rightarrow(0,+\infty)$ satisfies $\lim _{\epsilon \rightarrow 0} \eta_{2}(\epsilon) / \epsilon^{2}=$ 0 , then $\epsilon \mapsto u^{\epsilon, \eta_{2}(\epsilon)}$ is a one-parameter tracking control law for $\gamma_{\mathrm{ref}}$.

Proof. The hypotheses of the corollary and the expressions of the controls appearing in Theorem 3.3 guarantee that the oscillatory controls are as follows:

$$
\begin{aligned}
& u_{\mathrm{osc}, a}(\tau, t) \in \operatorname{span}_{\mathbb{R}}\left\{\varphi_{j_{0}}(\tau)\right\}, \\
& u_{\mathrm{osc}, a}^{\epsilon_{1}}(\tau, t) \in \operatorname{span}_{\mathbb{R}}\left\{\varphi_{j_{0}}(\tau), \frac{1}{\epsilon_{1}} \varphi_{j_{1}}\left(t / \epsilon_{1}\right) \varphi_{j_{2}}(\tau)\right\},
\end{aligned}
$$

where $j_{0}, j_{1}, j_{2}$ vary in $\mathbb{N}$.

Thus the integrals of all the terms $\mathscr{T}$ that appear in the proof of Theorem 5.4 only contain product of trigonometric functions, more specifically cosines and sines and converge to zero uniformly with respect to $t$ as $\epsilon$ goes to zero if $\eta_{2}$ is such that $\lim _{\epsilon \rightarrow 0} \eta_{2}(\epsilon) / \epsilon^{2}=0$.

\subsubsection{Numerical simulation: submarine}

In this section, we illustrate the method to obtain a one-parameter control law in a concrete situation that fulfills the assumptions in Corollary 5.6. The algorithm described in Section 5.2 has been implemented with Scilab.

For our example, we have the submarine presented in Section 1.1.2 whose dynamics are given by (18) and (19). We consider the same kind of inertia matrix as in Section 1.1.2, taking

$$
J_{1}=1, \quad J_{3}=3, \quad M_{1}=1, \quad M_{2}=2, \quad M_{3}=3 .
$$

We recall that in the case under consideration Theorem 3.3 cannot be applied. However, our method provides a one-parameter control law that solves the tracking problem. The trajectory to be tracked is given by

$$
r(t)=(-t,-t,-t), \quad A_{33}(t)=1, \quad t \in[0,1]
$$

with initial condition $r(0)=(0,0,0), A(0)=\mathrm{Id}$, being Id the identity $3 \times 3$ matrix, $\Pi(0)=(0,0,0)$, $P(0)=(-1,-2,-3)$. Thus there are degrees of freedom in the attitude of the submarine, but the target position of the center of the submarine is fully determined.

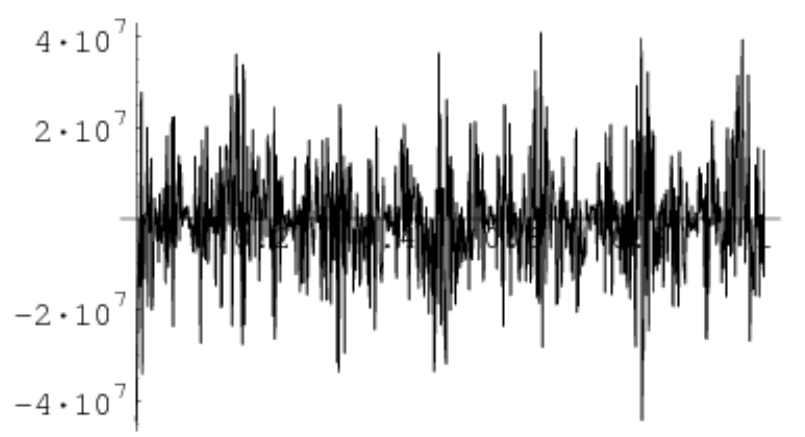

Figure 1: The control law $u_{1}^{\epsilon, \eta_{2}(\epsilon)}$.

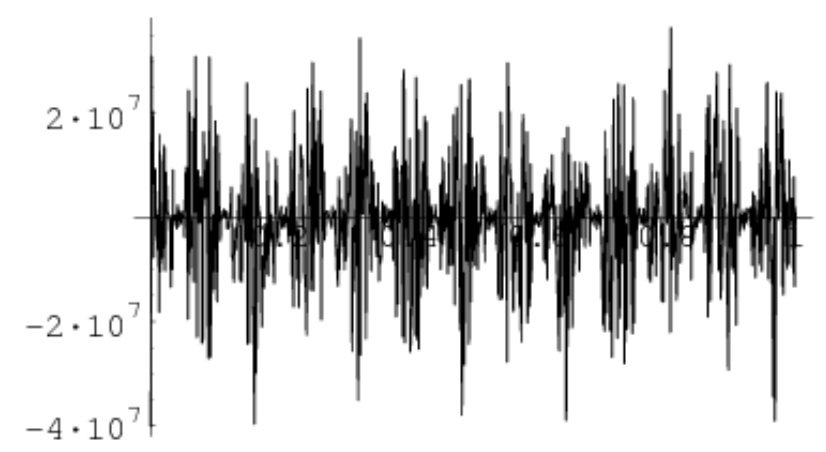

Figure 2: The control law $u_{2}^{\epsilon, \eta_{2}(\epsilon)}$.

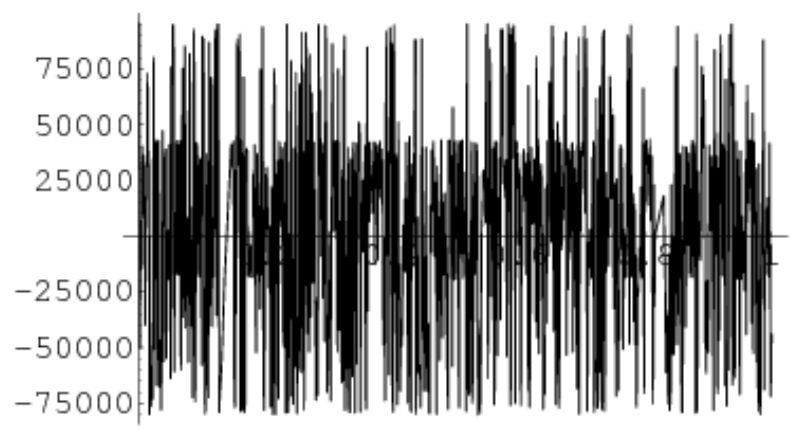

Figure 3: The control law $u_{3}^{\epsilon, \eta_{2}(\epsilon)}$. 
In this implementation we take $\eta_{2}(\epsilon)=\epsilon^{2.5}$ because the considered reference trajectory satisfies the hypotheses of Corollary 5.6.

First, we compute the one-parameter control laws $u_{1}^{\epsilon_{1}, \epsilon_{2}}, u_{2}^{\epsilon_{1}, \epsilon_{2}}$ and $u_{3}^{\epsilon_{1}, \epsilon_{2}}$ as described in the proof of Theorem 5.4. Then we fix $\epsilon=1 / 39 \approx 0.0256$, so that $\eta_{2}(\epsilon) \approx 0.0001$. The corresponding control laws are represented in Figures 1, 2 and 3. By construction, the controls are highly oscillatory.

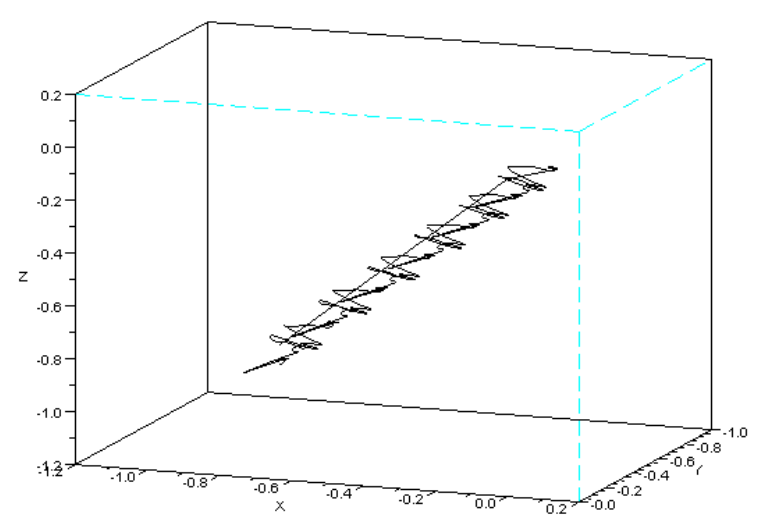

Figure 4: Evolution of the position of the center of the submarine with respect to time. The target trajectory is the non-oscillating curve.

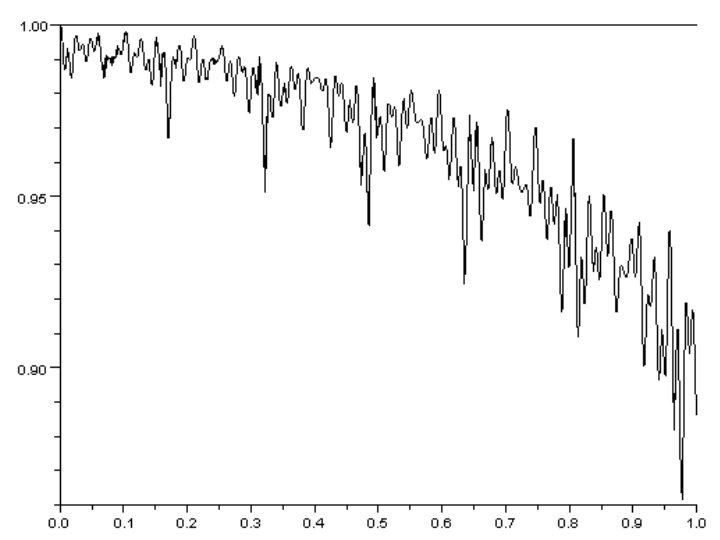

Figure 5: Evolution of $A_{33}$ entry of the attitude matrix with respect to time. The target trajectory is the non-oscillating curve.

Then we integrate the dynamics of the system using the numerical integrator stiff included in Scilab. As a result, Figures 10 and 5 show that the target trajectory, corresponding with the non-oscillating line, is tracked by the oscillating curve. The error of the approximation, computed by the supremum distance, is $\mathrm{d}\left(\left(r_{\mathrm{ref}}(t), A_{33, \mathrm{ref}}(t)\right),\left(r(t), A_{33}(t)\right)\right) \approx$
0.1903.

\section{Conclusions}

The previously known sufficient conditions for tracking were given in terms of finite sets of vector fields, as reviewed in Section 3. Here we have constructed a sequence of infinite family of vector fields that defines a sequence of convex cones suitable for characterizing trackability (Theorem 4.4 and Corollary 4.5). Different convex cones, (9) and (16), have been considered. Under additional assumptions, using the cones in (16) and a particular sequence of finite families of vector fields, it is possible to recover the sufficient conditions for tracking already known in the literature [5], see Corollary 4.7. However, our constructions not only recover the previously known results, but they also extend them, as shown in Section 4.1 .

The sequence of families of vector fields in Corollaries 4.5 and 4.7 are also suitable for constructing a one-parameter tracking control law (Theorems 5.2 and 5.4). It remains as future work to generalize the construction of one-parameter tracking control laws when the sets (9), that include the closure, are considered.

Another future research line is the study of the complexity for the control-affine systems considered in this work. Apart from tracking non-admissible trajectories, one could impose more requirements on the solution to the tracking problem, as for instance, to save energy. The complexity provides a good tool to formulate this kind of problems and, so far, has been only studied for control-linear systems [7, 8].

\section{Acknowledgements}

The first author acknowledges the financial support of Comissionat per a Universitats i Recerca del Departament d'Innovació, Universitats i Empresa of Generalitat de Catalunya in the final preparation of this paper.

\section{References}

[1] R. Abraham and J. E. Marsden, Foundations of Mechanics. Benjamin/Cummings Publishing Co. Inc. Advanced Book Program, Reading, Mass., second edition, 1978. 
[2] A. Agrachev and Y. L. Sachkov. Control Theory from the Geometric Viewpoint, volume 87 of Encyclopaedia of Mathematical Sciences. SpringerVerlag, Berlin, 2004.

[3] D. C. Biles, Continuous dependence of nonmonotonic discontinuous differential equations, Trans. Amer. Math. Soc., 339(2)(1993), 507524.

[4] A. Bressan and Z. Wang, On the controllability of Lagrangian systems by active constraints, J. Differential Equations (2009), doi:10.1016/j.jde.2009.01.014.

[5] F. Bullo and A. D. Lewis, Geometric Control of Mechanical Systems. Modeling, analysis and design for simple mechanical control, Texts in Applied Mathematics 49, Springer-Verlag, New York-Heidelberg-Berlin 2004.

[6] T. Chambrion and M. Sigalotti, Tracking control for an ellipsoidal submarine driven by Kirchhoff's laws. IEEE Trans. Automat. Control 53(1)(2008), 339-349.

[7] J. P. Gauthier and V. Zakalyukin, On the motion planning problem, complexity, entropy, and nonholonomic interpolation, J. Dyn. Control Syst. 12(3)(2006), 371-404.

[8] F. Jean, Complexity of nonholonomic motion planning. Internat. J. Control 74(8)(2001), 776-782.

[9] H. Lamb, Hydrodynamics. Reprint of the 1932 sixth edition. Cambridge Mathematical Library. Cambridge University Press, Cambridge, 1993

[10] J. M. Lee, Introduction to Smooth Manifolds, volume 218 of Graduate Texts in Mathematics. SpringerVerlag, New York, 2003.

[11] W. Liu and H. J. Sussmann, Continuous dependence with respect to the input of trajectories of control-affine systems, SIAM J. Control Optim., 37(3)(1999), 777-803.

[12] S. Martínez and J. Cortés, Motion control algorithms for simple mechanical systems with symmetry, Acta Appl. Math. 76(3)(2003), 221264.

[13] L. M. Milne-Thomson, Theoretical hydrodynamics, 4th ed. The Macmillan Co., New York, 1960.
[14] A. Munnier, Locomotion of deformable bodies in an ideal fluid: Newtonian versus Lagrangian formalisms, Journal of Nonlinear Sciences, to appear.

[15] K. Y. Pettersen and H. Nijmeijer, Underactuated ship tracking control: theory and experiments. Internat. J. Control 74(14) (2001), 1435-1446.

[16] J. A. Sanders, F. Verhulst, J. Murdock, Averaging methods in nonlinear dynamical systems. Second edition. Applied Mathematical Sciences, 59. Springer, New York, 2007. 